\title{
Where are the AGS snakes?
}

\section{F. Méot, R. Gupta, H. Huang, N. Tsoupas}

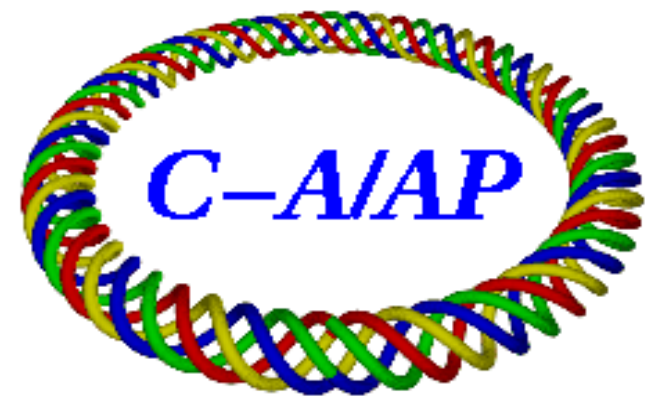

\section{Collider-Accelerator Department Brookhaven National Laboratory \\ Upton, NY 11973}

Notice: This document has been authorized by employees of Brookhaven Science Associates, LLC under Contract No. DE-AC02-98CH10886 with the U.S. Department of Energy. The United States Government retains a nonexclusive, paid-up, irrevocable, world-wide license to publish or reproduce the published form of this document, or allow others to do so, for United States Government purposes. 


\title{
Where are the AGS snakes?
}

\author{
F. Méot, R. Gupta, H. Huang, N. Tsoupas
}

BNL C-AD, Upton, LI, NY 11973

July 20th, 2013

\begin{abstract}
This note re-visits fields, particle motion, and spin precession in the AGS snakes, a work undertaken in preparation to the polarized proton RHIC Run 13 (February-June 2013), and in preparation of future polarized helion runs. These investigations include re-computation of 3-D OPERA field maps of the snakes and use stepwise ray-tracing tools and methods. There is a series of sub-products of this study, amongst others, the transport of the spin stable axis from AGS to RHIC X and Y kicker exit, the appropriate settings of the cold snake when changing its strength, cold snake settings for polarized helion programs, and in addition, a series of high accuracy 3-D field maps has been produced, in view of long-term motion and spin tracking in the AGS for the sake of polarization simulations to come.
\end{abstract}




\section{Contents}

1 Introduction 13

2 AGS snake angles, proton $\quad \ldots$

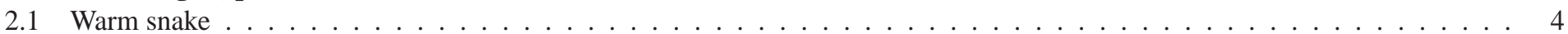

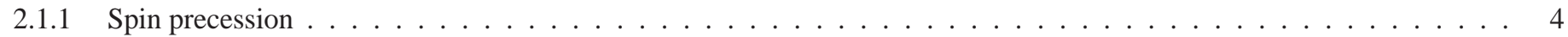

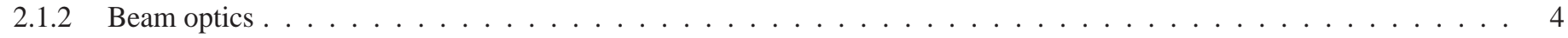

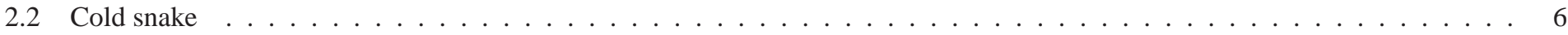

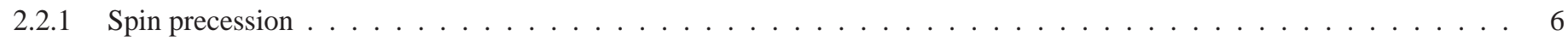

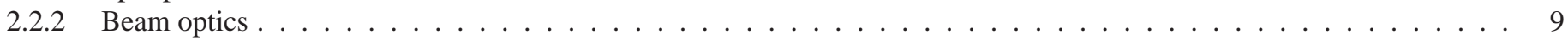

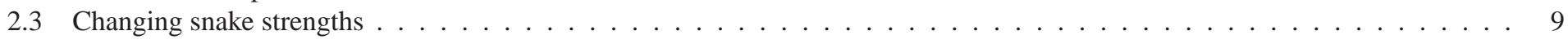

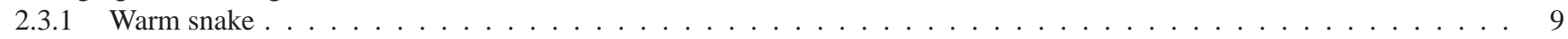

2.3 .2 Cold snake $\ldots \ldots \ldots \ldots \ldots \ldots \ldots \ldots \ldots$

3 Linear combination of helix and solenoid 3-D maps 10

4 Transport of $\vec{n}_{0}$, from H10 septum to RHIC kickers

5 Helion 13

6 Conclusion 15

Appendix 16

A Spin precession in warm snake 16

B Spin precession in cold snake $\quad 18$

C Cold snake, linear combination of helix and solenoid field maps 18

D Cold snake, cancelling $\int B_{z} d s$ in presence of $68.2 \%$ or $71 \%$ helix fields 19 


\section{Introduction}

A series of questions have arisen, in preparation to the polarized protons RHIC Run 13, and of future polarized helion runs, regarding the field settings as well as the spin precession angles in the AGS warm and cryogenic helical partial snakes. This is the main reason for the present studies, which consist essentially in re-visiting and clarifying various parameters of the snakes, and assessing their optimum settings. This work also led to a re-assessment of the periodic stable spin precession axis in the AGS at extraction, and of its transport to RHIC ; this is briefly reported here and will be subject to a detailed companion Tech. Note.

New OPERA field maps of the cold snake have been computed for the purpose of these studies, with various helix and/or solenoid current settings, they have been archived in dedicated folders in

$$
\text { /rap/lattice_tools/zgoubi/AgsZgoubiModel/snakeFieldMaps/coldSnake/RGFiles_Mar2013 }
$$

The other, original, OPERA field maps of the cold snake have been archived in additional dedicated folders in

$$
\text { /rap/lattice_tools/zgoubi/AgsZgoubiModel/snakeFieldMaps/coldSnake/ }
$$

No new warm snake OPERA maps have been computed up to now, although it is planned to do so, as discussed in due place in this document. All presently available maps, including the one used here, have been archived in

$$
\text { /rap/lattice_tools/zgoubi/AgsZgoubiModel/snakeFieldMaps/warmSnake/ }
$$

"README" files in these various folders give a guidance as to the respective properties and usage of the various maps.

These field maps are instrumental in the modeling of the AGS [1] using the ray-tracing code Zgoubi [2], they are part of the AGS optical sequence. In order to allow possible further checks and investigations, the main templates of the optics files as used in the present studies of spin rotation angles have been archived, for respectively the proton snake conditions and the helion conditions, in the folders

$$
\begin{gathered}
\text { /rap/lattice_tools/zgoubi/AgsZgoubiModel/snakeFieldMaps/rotationAngle/proton } \\
\text { /rap/lattice_tools/zgoubi/AgsZgoubiModel/snakeFieldMaps/rotationAngle/3He }
\end{gathered}
$$

where they can be copied from. A "README" guide can be found there, as well.

The code executable can be found at

$$
\text { /rap/lattice_tools/zgoubi/zgoubi }
$$

An extensive logbook regarding these investigations has been maintained, which the present Tech. Note abundantly refers to, it is archived at

$$
\text { /rap/lattice_tools/zgoubi/AgsZgoubiModel/snakeFieldMaps/rotationAngle/Log_130103/slides.pdf }
$$

An additional benefit of this study is that new field maps with denser mesh and longer extent have eventually been computed. The denser mesh brings better precision on field interpolation along particle trajectories by Zgoubi, and thus on particle and spin motion, whereas the longer extent of the maps allows reaching appropriately low field amplitudes at map ends, in the order of 10ppm of the field value at the center of the magnet. Both effects improve the long-term multi-turn tracking accuracy. Worth stressing, in passing : the principle of multiturn tracking using field maps is fully viable, as proven in modelling the AGS in Zgoubi with the best magnet models available, namely, in addition to the two snake 3-D OPERA field maps, representing the 240 main magnets with as many field maps derived from two sets of 2-D, mid-plane magnetic field measurements, still allowing efficient and accurate 1000s of turns motion and spin tracking, Ref. [3].

\section{AGS snakes}

Their role is to $z$-rotate the spin at each passage, by an angle $\phi \approx 10-12$ degrees, warm snake case, or $\phi \approx 20-28$ degrees, cold snake, proton case (about twice as much for helion). This allows overcoming depolarizing resonances, of both imperfection type $Q_{s p}=$ integer, and vertical intrinsic type $Q_{s p} \pm Q_{y}=$ integer [4, 5], according to the following principles :

Under the effect of the snakes during the acceleration cycle, spins undergo resonant flipping each time the spin tune satisfies $Q_{s p}=$ integer (41 times, from $G \gamma=4.5$ at injection to 45.5 at extraction), whereas placing the vertical betatron tune $Q_{y}$ in the forbidden spin tune gap integer $\pm \phi / 2 \pi$, as resulting from $\cos \pi Q_{s p}=\cos \frac{\phi}{2} \cos \pi G \gamma$, eliminates vertical intrinsic resonances. Non-linear snake resonances $Q_{s p} \pm l Q_{y}=$ integer, $l \geq 2$, present in the spin tune forbidden gap, are avoided by adjusting $Q_{y}$ with a margin that depends on the strength of the local linear resonance [6].

On the other hand the stable spin precession direction $\vec{n}_{0}$ is in general at a (small) angle to the vertical around the ring. This drives horizontal intrinsic spin resonances, $Q_{s p} \pm Q_{x}=$ integer, their effect is minimized by 80 local tune jumps, using a pair of dedicated, fast quadrupoles [7]. AGS optics in addition includes coupling sources (e.g., main magnet defects, the snakes themselves at low energy), which also excites coupling resonances $Q_{s p} \pm Q_{x}=$ integer.

The extraction $G \gamma$ value ( $G \gamma=45.5$ in recent RHIC polarized proton runs) is chosen at the optimum match between the periodic $\vec{n}_{0}$ at the extraction septum H10 in the AGS, and the vertical periodic $\vec{n}_{0}$ at the injection kicker in RHIC. The transport from the former to the latter is ensured by the AtR (AGS to RHIC), a non-planar beam line [8]. An update of these AtR data using the new snake field maps is given in a companion paper [9].

Thus, accurate control of beam polarization in the AGS, of its transport along the acceleration cycle, and of its delivery, the closest possible to vertical, at RHIC-blue (down the "X" branch of the AtR) and RHIC-yellow ("Y" branch) injection kickers, require an accurate knowledge of the snake fields and of their settings. 


\section{AGS snake angles, proton}

Extensive numerical simulations have been performed, including the computation of a new series of OPERA field maps of the cold snake. Orbit helices, spin precession, and other snake parameters resulting from these simulations may be compared in a detailed way to the initial studies [10]-[15] using the material available in Ref. [16].

Prior to computing the spin precession, the helical orbit is first, systematically, centered on the snake axis at the $G \gamma$ value of concern, using the fitting procedure in Zgoubi. The methods and software tools and files used are discussed in Ref. [16], templates of the latter may be found in the above mentioned archive folders.

Note - In polarized proton AGS operation, in the case of the cold snake, a local orbit bump using AGS main magnets back-leg windings partially compensates the orbit defect introduced by the snake, up to $300 \mathrm{~ms}$ about after injection, $G \gamma \approx 13.5$, beyond what the orbit defect is considered marginal and the correction cancelled. In the case of the warm snake, since the helix field and thus the induced orbit defect are smaller, a local compensation bump is considered un-necessary. As a consequence, the orbit helix in the snakes during actual AGS operation may not be strictly centered in the snakes, by contrast to the working hypotheses, here. Possible effects of such mis-centering are not part of the present study.

\subsection{Warm snake}

The AGS warm snake, installed in 2004 [17], located in the E20 straight section, is a normal conducting helical dipole with double pitch structure (0.4/0.195/0.4 deg/m over 0.39/1.32/0.39 m, entrance/body/exit) designed to ensure minimized orbit defect [4].

The same 3-D OPERA field map as investigated in Ref. [15] is used here, it is known and archived under the name

"table55.tab"

It was computed for the nominal operation conditions, namely, $1.53 \mathrm{~T}$ field modulus at the center of the magnet, 2540 Amp coil current, for additional details see Ref. [17].

Orbit and spin tracking data, as obtained from ray-tracing, are detailed in Figs. 2-6.

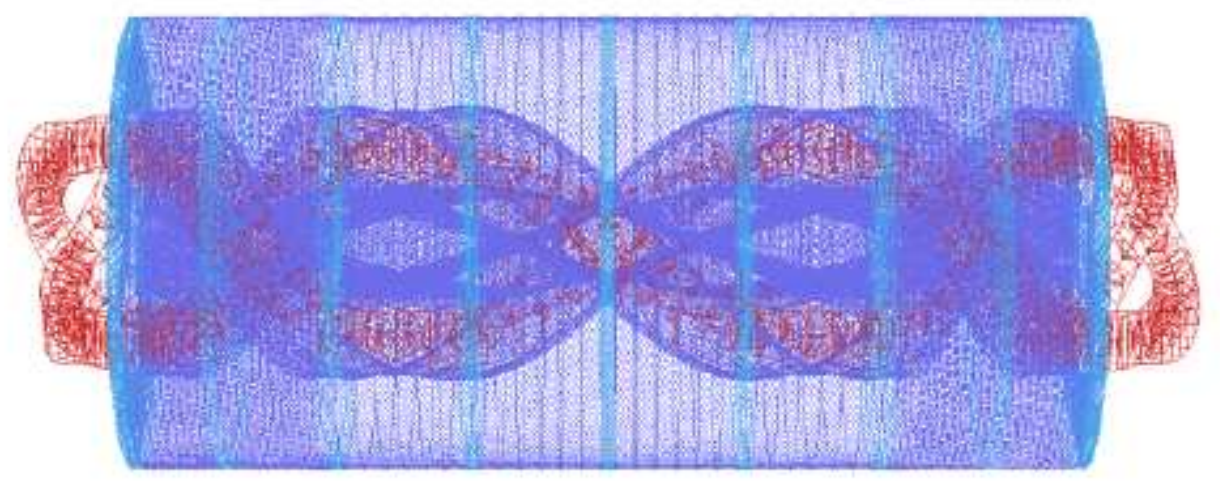

Figure 1: 3-D OPERA model of the warm snake [17].

\subsubsection{Spin precession}

Spin behavior, from ray-tracing through the 3-D OPERA map, is displayed in Figs. 5, 6. The characteristics in Tab. 1, column 3 are obtained.

The spin precession is interpolated at better than $210^{-3}$ accuracy (relative to actual tracking outcomes) from $G \gamma=4.5$ and beyond, and with $B$ in the vicinity of $B_{0}$, by, see Fig. 6 [16, Sec. 1.1],

$$
\begin{gathered}
\mu(B, G \gamma)[\text { deg. }]=\left(B / B_{0}\right)^{2}\left[C_{0}+C_{1} / G \gamma+C_{2} /(G \gamma)^{2}\right] \\
\text { with } B_{0}=1.5333 \text { Tesla, } C_{0}=10.578, C_{1}=-1.284, C_{2}=34.60
\end{gathered}
$$

Checking data from earlier studies [15] was part of the motivations for the present work, discrepancies have been found, they are briefly addressed in App. A, details in Ref. [16, Sec. 1.2].

\subsubsection{Beam optics}

The first order mapping of the snake is rigidity dependent. It is computed from particle trajectories taken paraxial to the $G \gamma$-dependent helical orbits across the magnet. By contrast with spin precession, today's particle optics data come out to be in good agreement with those established in detail in Ref. [15] and used in the MADX model of the AGS [18]. Some comparisons can be found in Ref. [16, Sec. 1.2]. 


\section{Warm snake. Motion, field, spin precession}
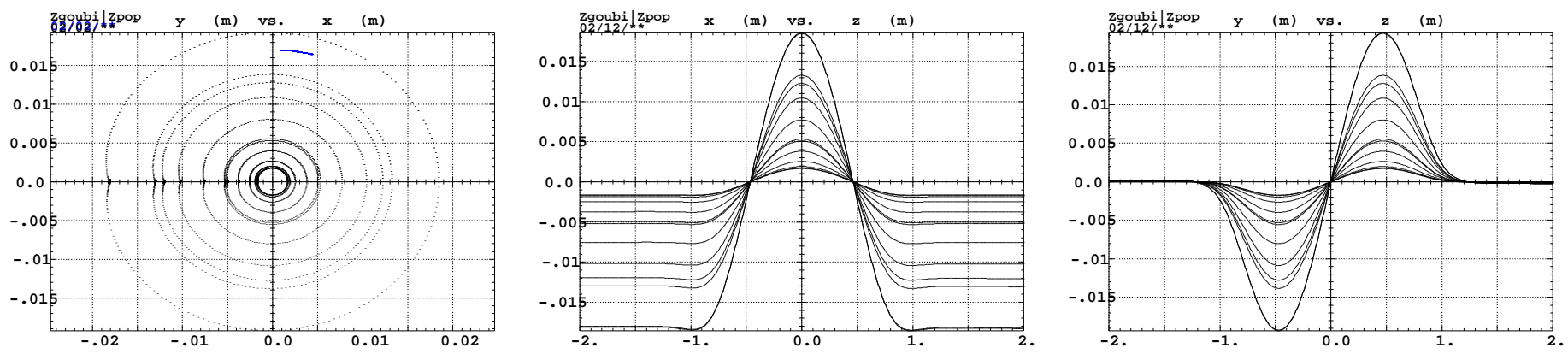

Figure 2: Projection of helical orbits with $G \gamma \in[4.5,50]$ onto, left : transverse plane $(x, y)$ (positive $x$ is toward the center of the AGS) ; center : horizontal plane ; right : vertical plane. Starting position in this simulation is, left plot, $\left(x=x_{m i n}, y=0\right)$. In addition, left plot : the short arc, top of first quadrant, represents the spin $z$-rotation on these various orbits, details in Fig. 5.

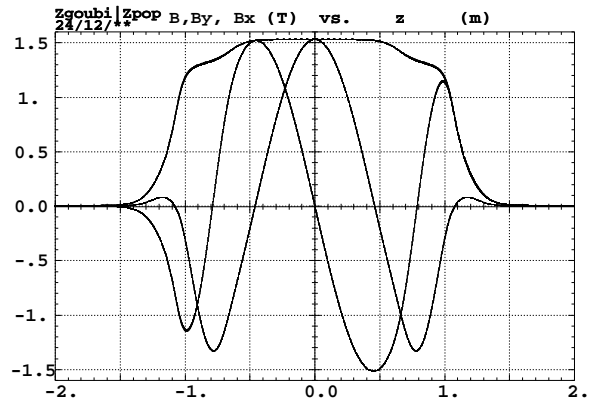

Figure 3: Field along orbits, left : $|\vec{B}|, B_{x}, B_{y}$, right : $B_{z}$.

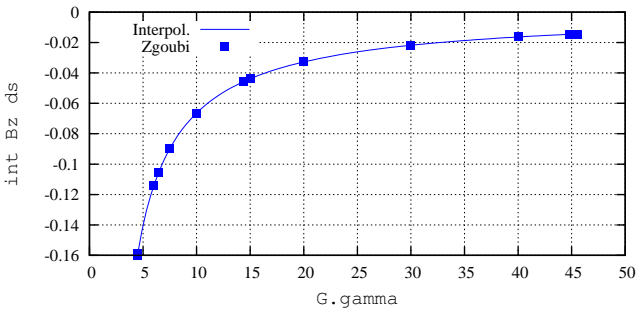

Figure 4: Integral of longitudinal field component along the orbit (squares) and spline interpolation (solid line).
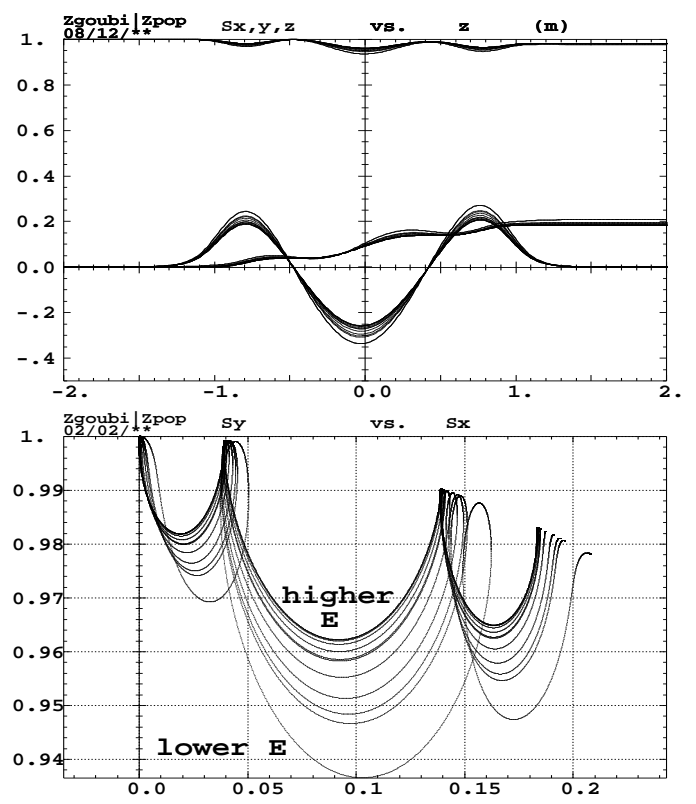

Figure 5: Top : Spin components along helical orbits. Bottom : spin motion in the $\left(S_{x}, S_{y}\right)$ plane, initial position is vertical, precession is $G \gamma$ dependent.
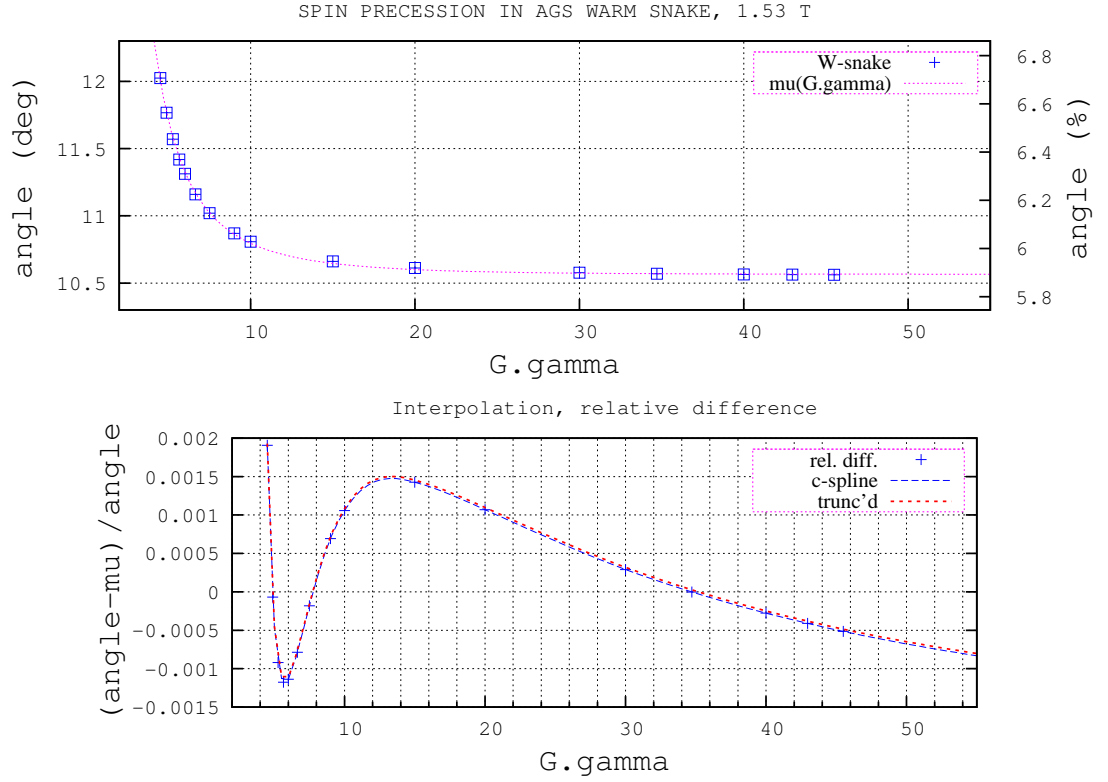

Figure 6: Top : value of spin precession angle in the warm snake, at various $G \gamma$ values, and interpolation function with truncated $\mathrm{B} 0, \mathrm{C} 0-\mathrm{C} 2$ data of Eq. 1. Bottom : relative difference between angle from tracking ("angle") and from interpolation $\mu(G \gamma)$; the "trunc'd" curve is for the truncated values of $\mathrm{C} 0-\mathrm{C} 2$ of Eq. 1, the "rel. diff." curve, for comparison, is at computer precision on matched $\mathrm{C} 0-\mathrm{C} 2$ data. 
Table 1: Characteristics of the warm and cold snakes, polarized proton RHIC Run 13 parameters. The warm snake field map is the original one, 2003 about [15]. The cold snake ones, in both 152 Amp, 81.2 Amp and zero solenoid current cases, result from new, 2013, OPERA computations. The zero current case is documented for all practicle purposes.

\begin{tabular}{|c|c|c|c|c|c|}
\hline \multirow[b]{2}{*}{$\begin{array}{l}\text { Helix coil current } \\
\text { (relative to } 350 \mathrm{Amp} \text { ) }\end{array}$} & \multicolumn{2}{|r|}{ Warm snake ${ }^{(a)}$} & \multicolumn{3}{|c|}{ Cold snake } \\
\hline & $\begin{array}{l}\mathrm{A} \\
\%\end{array}$ & 2540 & $\begin{array}{c}248.5 \\
(71)\end{array}$ & $\begin{array}{c}248.5 \\
(71)\end{array}$ & $\begin{array}{c}248.5 \\
(71)\end{array}$ \\
\hline Solenoid current & A & - & 152 & 81.2 & 0 \\
\hline$|\vec{B}|$ at helix center, $B_{00}$ & $\mathrm{~T}$ & 1.5309 & 2.2402 & 2.2598 & 2.2211 \\
\hline $\int_{\text {axis }}|\vec{B}(z)| d z$ & T.m & 3.2276 & 4.4629 & 4.5282 & 4.4467 \\
\hline Straight mag. $\mathcal{L}=\frac{\int_{\text {axis }}|\vec{B}| d z}{B_{00}}$ & $\mathrm{~m}$ & 2.1083 & 1.9922 & 2.0038 & 2.0020 \\
\hline Max. $|\vec{B}(s)|$ on orbits, $G \gamma: 4.5 \rightarrow 50$ & $\mathrm{~T}$ & $1.5333 \rightarrow 1.5309$ & $\approx 2.239$ & $\approx 2.259$ & $2.233 \rightarrow 2.221$ \\
\hline $\int_{\text {orbit }} B_{z}(s) d s, G \gamma: 4.5 \rightarrow 50$ & T.m & $-0.16 \rightarrow-0.014$ & $\begin{array}{l}-0.04 \rightarrow+0.23 \\
(0 \text { at } G \gamma \approx 5.2)\end{array}$ & $\begin{array}{l}-0.17 \rightarrow+0.11 \\
(0 \text { at } G \gamma \approx 9)\end{array}$ & $-0.30 \rightarrow-0.02$ \\
\hline Orbit radius, $G \gamma: 4.5 \rightarrow 50$ & $\mathrm{~mm}$ & $19.2 \rightarrow 1.6$ & $23.4 \rightarrow 1.9$ & $23.4 \rightarrow 1.9$ & $23.6 \rightarrow 1.9$ \\
\hline Spin prec. $\phi, G \gamma: 4.5 \rightarrow 50$ & deg & $12.04 \rightarrow 10.56$ & $27.62 \rightarrow 20.0$ & $26.0 \rightarrow 20.4$ & $22.50 \rightarrow 19.5$ \\
\hline Snake strength & $\%$ & $6.69 \rightarrow 5.87$ & $15.34 \rightarrow 11.11$ & $14.44 \rightarrow 11.33$ & $12.5 \rightarrow 10.83$ \\
\hline
\end{tabular}

(a) Map name : "table55.tab", as used in Zgoubi input data files.

(b) Map name : "ags-full-coilv5-x06-rerun2-x071-sol-152a-nodal-x5y5z10mm.table” (ibid.).

(c) Map name : "ags-helical-solenoid-april2013-coilv5-x071-sol-81_2A-integral-x5y5z10mm.table” (ibid.).

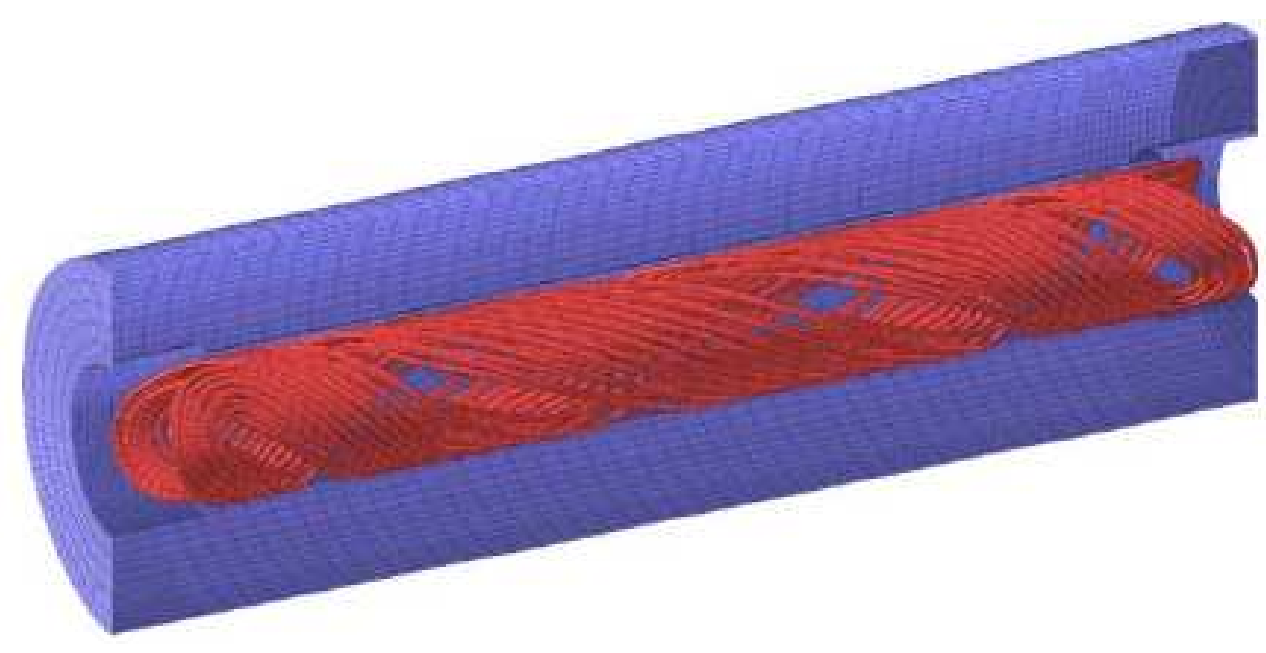

Figure 7: 3-D model of the cold snake, OPERA simulation.

\subsection{Cold snake}

The AGS cold snake, installed in 2005 and first operated in $2006[19,20]$, is located in the A20 straight section in the AGS. It is a superconducting helical dipole with double pitch structure $(0.392 / 0.2053 / 0.392 \mathrm{deg} / \mathrm{mm}$ over $0.393 / 1.154 / 0.393 \mathrm{~m}$, entrance/body/exit), which ensures minimized orbit defect. The device includes a $0.82 \mathrm{~m}$ long solenoid winding aimed at cancelling the longitudinal field integral $\int B_{z} d s$ experienced by the particles on the helical orbit, at $G \gamma=9$. It also includes a $0.3 \mathrm{~m}$ long, 0.008 T.m, vertical corrector at both ends.

Orbit and spin tracking data in the case of 152 Amp current in the $\vec{B}_{s}$ compensation solenoid, the actual setting in recent RHIC polarized proton runs, as obtained from ray-tracing, are detailed in Figs. 8-12.

It was realized in the course of the present study, that the compensation solenoid setting should instead be 81.2 Amp about for the present $71 \%$ helix (248.5 Amp helix current), or equivalently should be $(0.682 / 0.71)^{2} \times 81.2=74.9 \mathrm{Amp}$ in the case of, Run 13 settings, $68.2 \%$ helix (238 A). Orbit and spin tracking data in the case of a $81.2 \mathrm{Amp}$ current in the solenoid, which has the property of cancelling $\int B_{z} d s$ at $G \gamma=9$, are detailed in Figs. 13-17. It can be observed that such change in solenoid current is not innocent in terms of snake strength, it changes the spin precession by a sensible amount, larger at lower energy, cols. 4, 5 in Tab. 1 and Figs.12, 17.

\subsubsection{Spin precession}

Case of solenoid field 152 Amp - From ray-tracing through the 71\% helix and 152 Ampere solenoid 3-D OPERA map

$$
\text { “ags-full-coilv5-x06-rerun2-x071-sol-152a-nodal-x5y5z10mm.table” }
$$

the characteristics in Tab. 1, column 4 are obtained. Spin behavior is displayed in Figs. 11, 12. 


\section{Cold snake Hlx71\%-Sol152Amp. Motion, field, spin precession}
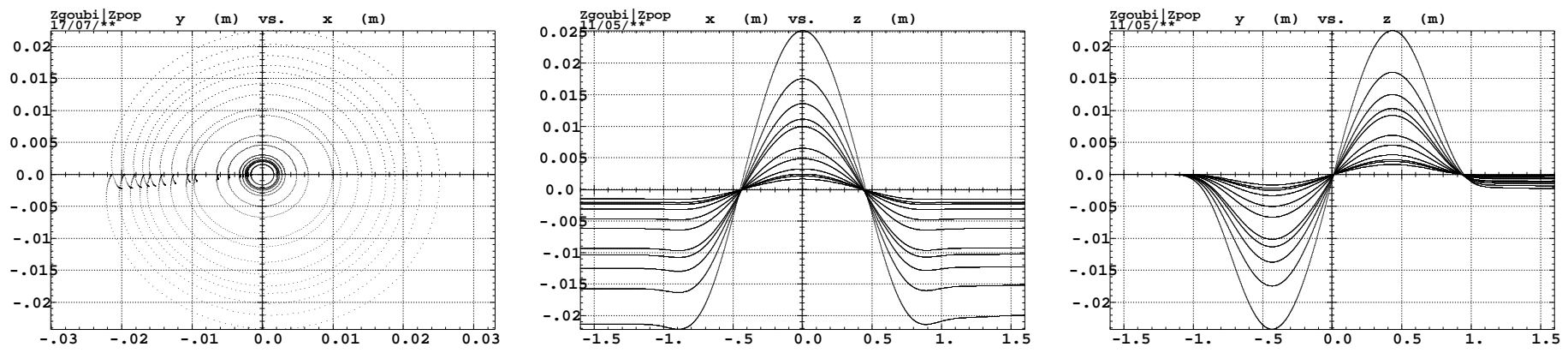

Figure 8: Projection of helical orbits with $G \gamma \in[4.5,50]$ onto, left : transverse plane $(x, y)$ (positive $x$ is toward the center of the AGS) ; center : horizontal plane ; right : vertical plane. Starting position in this simulation is, left plot, $\left(x=x_{\text {min }}, y=0\right)$.
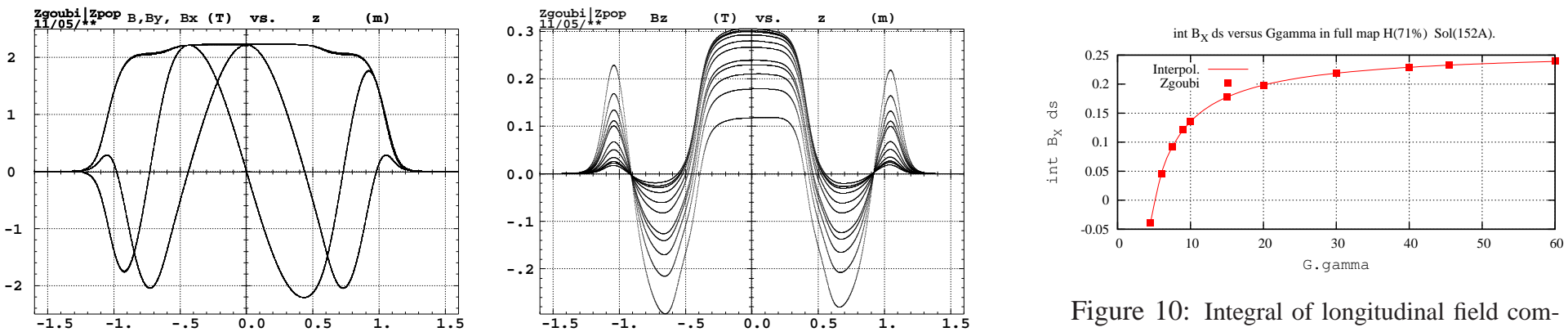

Figure 9: Field along orbits, left : $|\vec{B}|, B_{x}, B_{y}$, right : $B_{z}$.

Figure 10: Integral of longitudinal field component along the orbit (markers, from Zgoubi tracking) and interpolating spline (solid line).

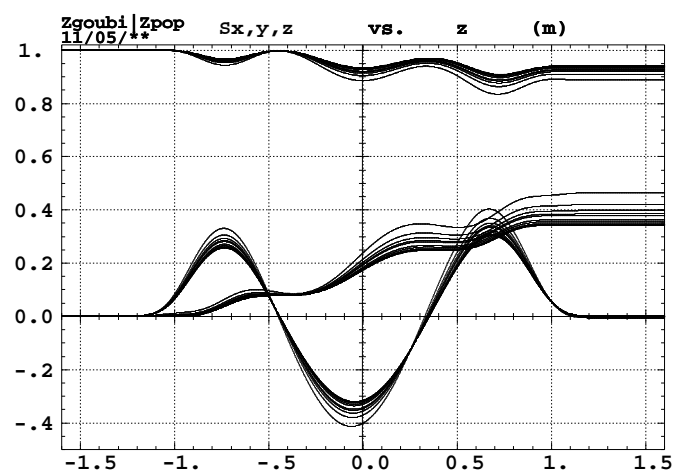

Figure 11: Spin components along helical orbits.
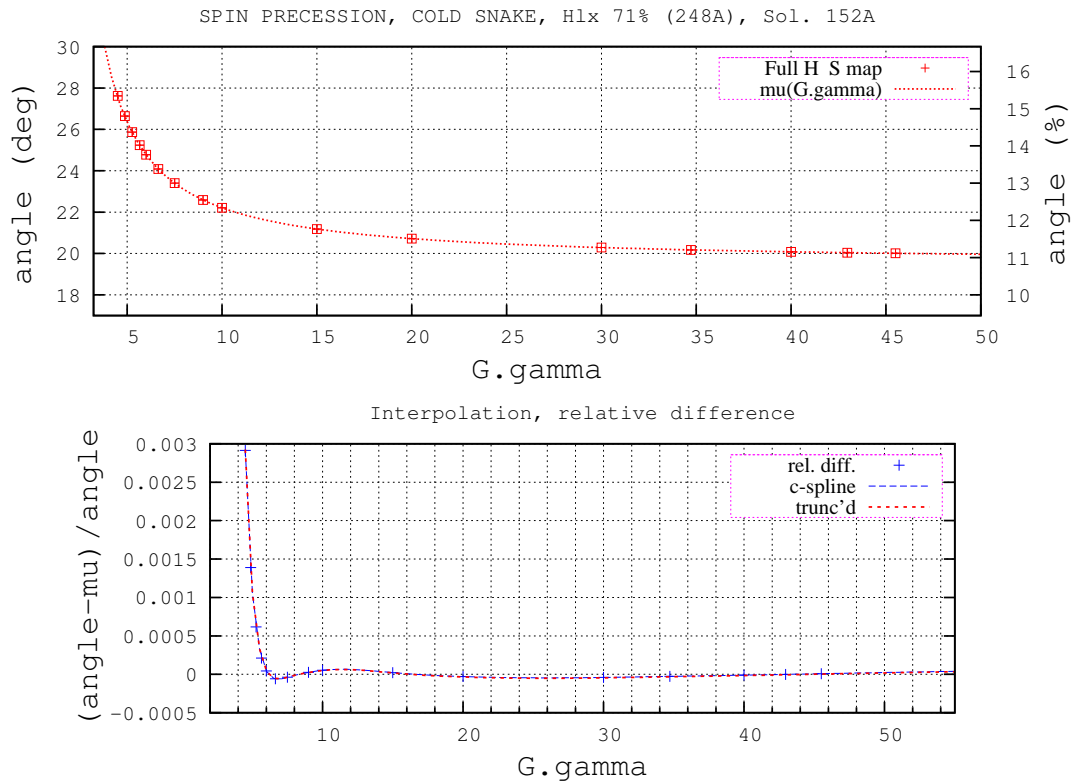

Figure 12: Top : value of spin precession angle in the cold snake, $71 \%$ helix and 152 Amp solenoid current, at various $G \gamma$ values, and interpolation function with truncated B0, C0-C3 data of Eq. 2. Bottom : relative difference between angle from tracking ("angle") and from interpolation $\mu(G \gamma)$; the "trunc'd" curve is for the truncated values of $\mathrm{C} 0-\mathrm{C} 3$ of Eq. 2, the "rel. diff." curve, for comparison, is at computer precision on matched $\mathrm{C} 0-\mathrm{C} 3$ data. 


\section{Cold snake Hlx71\%-Sol81Amp. Motion, field, spin precession}
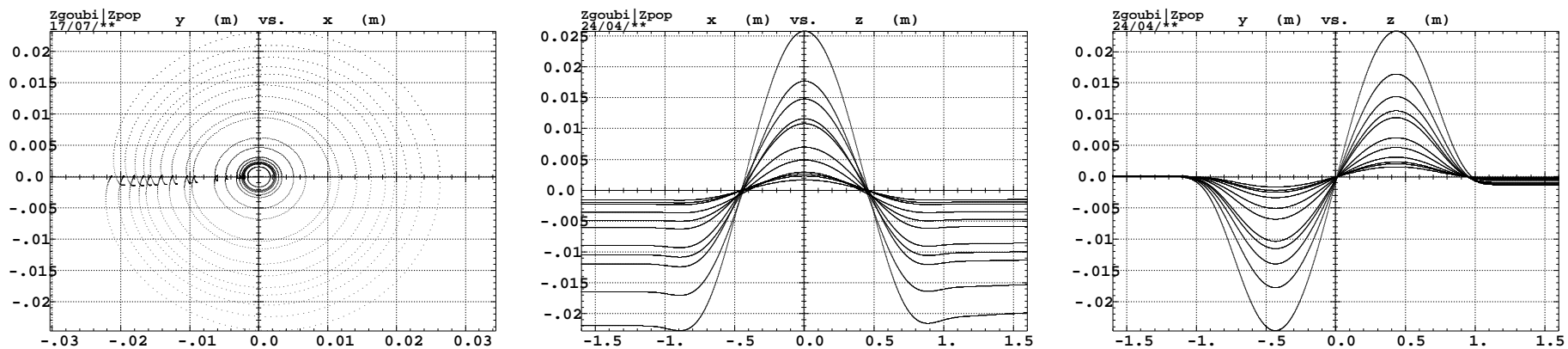

Figure 13: Projection of helical orbits with $G \gamma \in[4.5,50]$ onto, left : transverse plane $(x, y)$ (positive $x$ is toward the center of the AGS) ; center : horizontal plane ; right : vertical plane. Starting position in this simulation is, left plot, $\left(x=x_{\text {min }}, y=0\right)$.
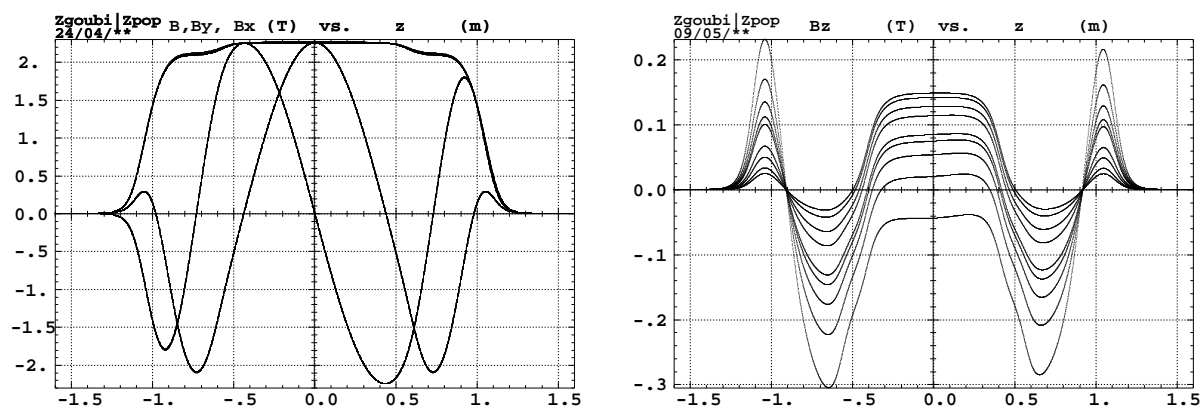

Figure 14: Field along orbits, left : $|\vec{B}|, B_{x}, B_{y}$, right : $B_{z}$.

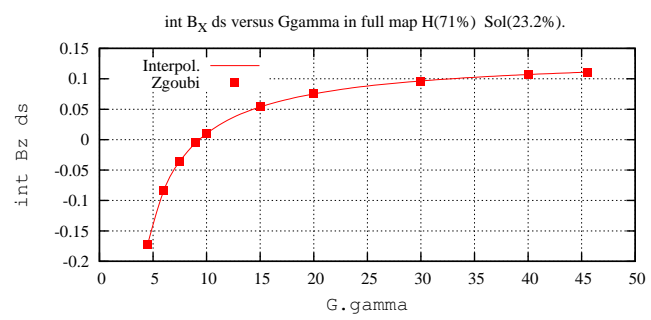

Figure 15: Integral of longitudinal field component along the orbit, it vanishes at $G \gamma \approx 9$. Square markers from Zgoubi tracking and interpolating spline (solid line).

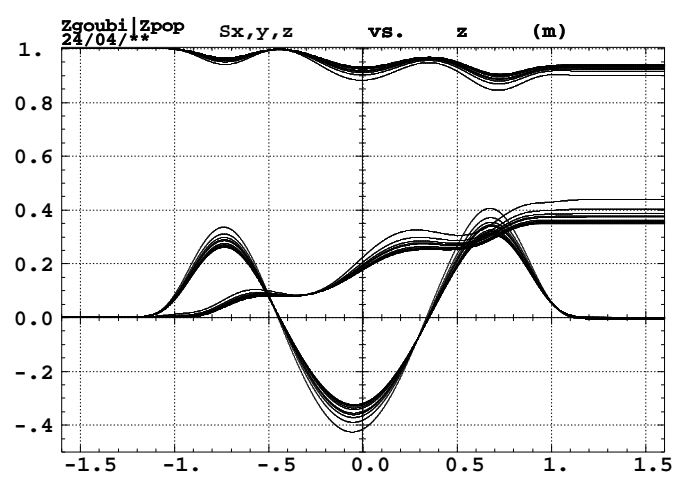

Figure 16: Spin components along helical orbits.
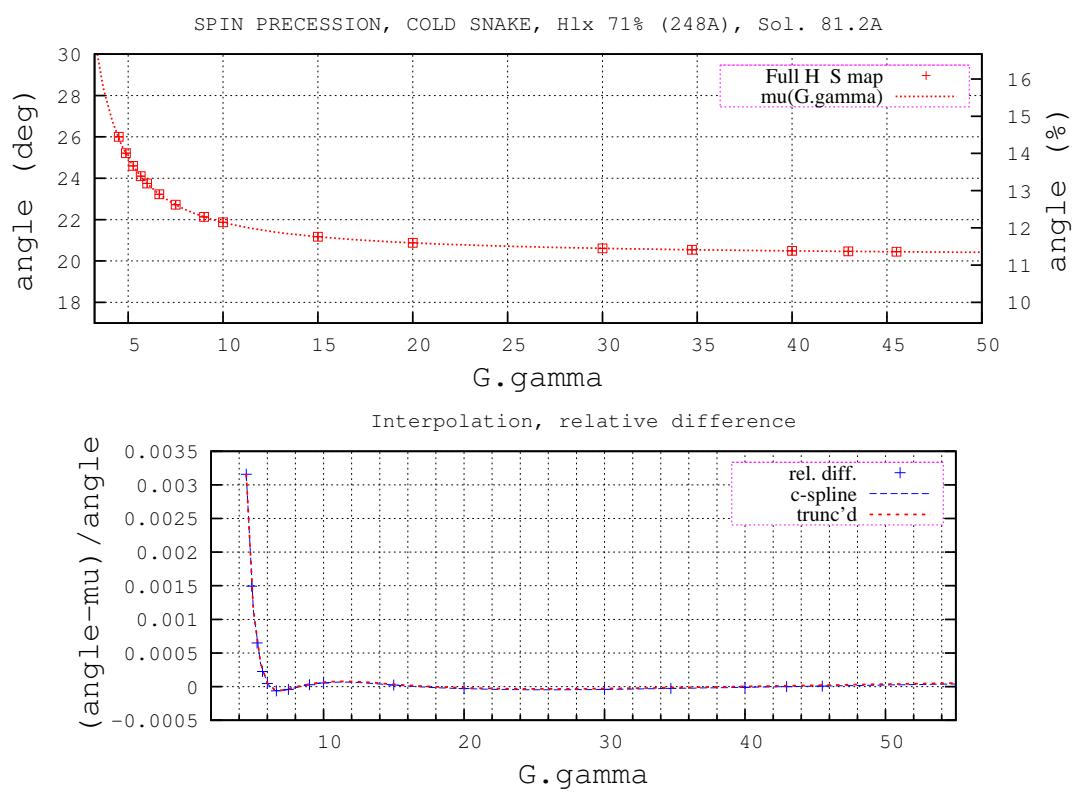

Figure 17: Top : value of spin precession angle in the cold snake, $71 \%$ helix and 81.2 Amp solenoid current, at various $G \gamma$ values, and interpolation function with truncated B0, C0-C3 data of Eq. 3. Bottom : relative difference between angle from tracking ("angle") and from interpolation $\mu(G \gamma)$; the "trunc'd" curve is for the truncated values of $\mathrm{C} 0-\mathrm{C} 3$ of Eq. 3, the "rel. diff." curve, for comparison, is at computer precision on matched $\mathrm{C} 0-\mathrm{C} 3$ data. 
The spin precession is interpolated at better than $310^{-3}$ accuracy (relative to actual tracking outcomes) from $G \gamma=4.5$ and beyond, and with $B$ in the vicinity of $B_{0}$, by [16, Sec. 4.1], Fig. 12,

$$
\begin{array}{r}
\mu(B, G \gamma)[\text { deg. }]=\left(B / B_{0}\right)^{2}\left[C_{0}+C_{1} / G \gamma+C_{2} /(G \gamma)^{2}+C_{3} /(G \gamma)^{3}\right] \\
\text { with } B_{0}=2.2387 \text { Tesla, } C_{0}=19.506, C_{1}=22.039, C_{2}=39.251, C_{3}=108.7
\end{array}
$$

A remark : comparisons with earlier studies [10]-[12] show discrepancies, this is briefly discussed in App. B, details in Ref. [16, Sec. 4.2]. It was found in particular that the longitudinal field integral $\int B_{z} d s$ along the helical trajectory does not cancel at $G \gamma=9$ with the RHIC Run 13 settings (152 A solenoid current for a 68.2\% helix field, which corresponds to 164 Amp about for a $71 \%$ helix). A solenoid current of about half the value was found to actually be the correct setting for that, namely, as obtained from tracking in combined field maps (details in App. D),

$$
\begin{aligned}
& \text { 76.0 A for a } 68.2 \%(238 \mathrm{~A}) \text { helix, } \\
& 82.5 \text { A for a } 71 \% \text { (248 A) helix, }
\end{aligned}
$$

Note the following :

(i) These solenoid currents are in the ratio of the square of the helix fields : $82.5 \times(68.2 / 71)^{2} \approx 76.1$, this is discussed in Sec. 2.3.2.

(ii) The 82.5 A solenoid current in the $71 \%$ helix case here, slightly differs from the value used elsewhere in the text, 81.2 Amp. The reason is simply that the latter results from an earlier, rougher assessment, however, tracking shows that the first value yields $\int B_{z} d s=1.810^{-5}$ T.m at $G \gamma=9$, whereas the second one yields $\int B_{z} d s=-2.310^{-3}$ T.m, both contributing in as marginal a way to coupling, anyway.

Case of solenoid field 81.2 Amp - From ray-tracing through the 71\% helix and 81.2 Ampere solenoid 3-D OPERA map

$$
\text { “ags-helical-solenoid-april2013-coilv5-x071-sol-81_2A-integral-x5y5z10mm.table” }
$$

the characteristics in Tab. 1, column 5 are obtained. Spin behavior is displayed in Figs. 16, 17.

The spin precession is interpolated at better than $310^{-3}$ accuracy (relative to actual tracking outcomes) from $G \gamma=4.5$ and beyond, and with $B$ in the vicinity of $B_{0}$, by [16, Sec. 4.4], Fig. 17,

$$
\begin{array}{r}
\mu(B, G \gamma)[\text { deg. }]=\left(B / B_{0}\right)^{2}\left[C_{0}+C_{1} / G \gamma+C_{2} /(G \gamma)^{2}+C_{3} /(G \gamma)^{3}\right] \\
\text { with } B_{0}=2.2588 \text { Tesla, } C_{0}=20.163, C_{1}=11.997, C_{2}=40.61, C_{3}=99.1
\end{array}
$$

\subsubsection{Beam optics}

The first order mapping of the snake is rigidity dependent. It comes out to be quite close to that established in detail in Ref. [13] and used in the MADX model of the AGS [18]. Matrix comparisons can be found in Ref. [16, Sec. 4.5].

\subsection{Changing snake strengths}

Changing the strength of the AGS snakes has an effect on the size of the spin tune gap, $\pm \phi / 2$, with $\phi$ the one-pass spin precession angle, and on the strength of the horizontal resonances via the orientation of the stable precession axis $\vec{n}_{0}$. This may have an effect on the polarization transmission along the acceleration cycle.

Changes in periodic $\vec{n}_{0}$ in the AGS would in addition change the orientation of $\vec{n}_{0}$ along the AtR line. One can therefore think of margins for optimization of the verticallity of $\vec{n}_{0}$ at RHIC X and Y kickers.

This makes it desirable to investigate changes in both warm and cold snakes.

\subsubsection{Warm snake}

Changes in the warm snake strength in the 10-20\% range may be computed using Eq. 1 as a first approach, neglecting the effects of saturation (the excitation curve is not too far from linear, with the present 1.53 Tesla field).

Further dedicated simulations including OPERA maps at higher or lower currents are however desirable. It is planned to work that out in the future.

Changing the warm snake would in addition require re-tuning the local compensation quadrupoles, in the low energy part of the AGS cycle. This is mainly a matter of dedicated ray-tracing simulations, following the methods in [16], using the appropriate OPERA maps and Zgoubi input data files as archived in the folders listed in Sec. 1. 


\subsubsection{Cold snake}

Changes in the cold snake are easier to compute since they do not require a new OPERA field map : as shown earlier and discussed further in Sec. 3, a linear combination of the helix and solenoid field maps (times a 1.016 correction factor in the case of solenoid field in 80 150 Amp range) can be used instead. However this requires taking into account the constraint of the cancellation of $\int B_{z} d s$ (at $G \gamma=9$ or else), which determines the solenoid current.

From the equations of the helical wiggler field [21],

$$
\begin{gathered}
B_{r}=2 B_{0}\left[I_{0}(k r)-(i / k r) I_{1}(k r)\right] \sin (\theta-k z) \\
B_{\theta}=\left(2 B_{0} / k r\right) I_{1}(k r) \cos (\theta-k z) \\
B_{z}=-2 B_{0} J_{1}(k r) \cos (\theta-k z)
\end{gathered}
$$

and from $r \propto B_{0}, J_{1}(k r) \approx k r$ to first order, one gets $B_{z} \propto B_{0}^{2}$. As a consequence the compensation solenoid field has to follow $B_{0}^{2}$ - as does the strength in the case of a full snake in first approximation, in passing [22] :

$$
\phi / \pi=2 \sqrt{1+\chi^{2}}-1 \stackrel{\chi^{2} \ll 1}{\approx} \chi^{2} \quad\left(\text { with } \quad \chi=\left(1+\frac{1}{G \gamma}\right) \frac{Z G c}{M[e V]} \frac{\int B d s}{2 \pi} \approx 0.1, \text { here }\right)
$$

Numerical data as obtained from Zgoubi tracking, regarding precession angle at $G \gamma=45.5$, proton, accounting for cancellation of $\int B_{z} d s$ at $G \gamma=9$, are displayed in Tab. 2 .

\begin{tabular}{|c|c|c|c|c|c|c|}
\hline \multirow{3}{*}{$\begin{array}{l}\text { 71\% Helix } \\
\text { Field } \\
\text { variation }\end{array}$} & \multicolumn{4}{|c|}{ Solenoid field cancels $\int B_{z} d s$ at $G \gamma=9$} & \multirow{2}{*}{\multicolumn{2}{|c|}{$\begin{array}{c}\text { Solenoid field is 0 } \\
\text { Snake angle at } G \gamma=45.5\end{array}$}} \\
\hline & \multicolumn{2}{|c|}{ Solenoid current } & \multicolumn{2}{|c|}{ Snake angle at $G \gamma=45.5$} & & \\
\hline & $(350 \mathrm{~A})$ & (A) & (deg.) & variation (\%) & (deg.) & variation (\%) \\
\hline$-10 \%$ & 0.188 & 65.75 & 16.61 & -18. & 15.90 & -18.6 \\
\hline$-5 \%$ & 0.209 & 73.28 & 18.47 & -9.5 & 17.68 & -9.5 \\
\hline 0 & 0.232 & 81.2 & 20.41 & 0 & 19.54 & 0 \\
\hline$+5 \%$ & 0.256 & 89.55 & 22.43 & +9.9 & 21.48 & +10 \\
\hline$+10 \%$ & 0.281 & 98.29 & 24.55 & +20.3 & 23.51 & +20.4 \\
\hline
\end{tabular}

Table 2: Varying the helix field in a $\pm 10 \%$ range around $71 \%$ helix setting. Case of polarized proton at $G \gamma=45.5$. Cols. 4-6 give the resulting precession angle : cols. 4,5 account for necessary solenoid strength to cancel $\int B_{z} d s$ at $G \gamma=9$, whereas cols. 6,7 assume pure helix.

\section{Linear combination of helix and solenoid 3-D maps}

During the present studies regarding the cold snake it has been verified and investigated further that the magnetic field in the useful region in the snake, in the case of solenoid field in 80 150 Amp range, can be obtained by a linear combination of the helix and solenoid field maps

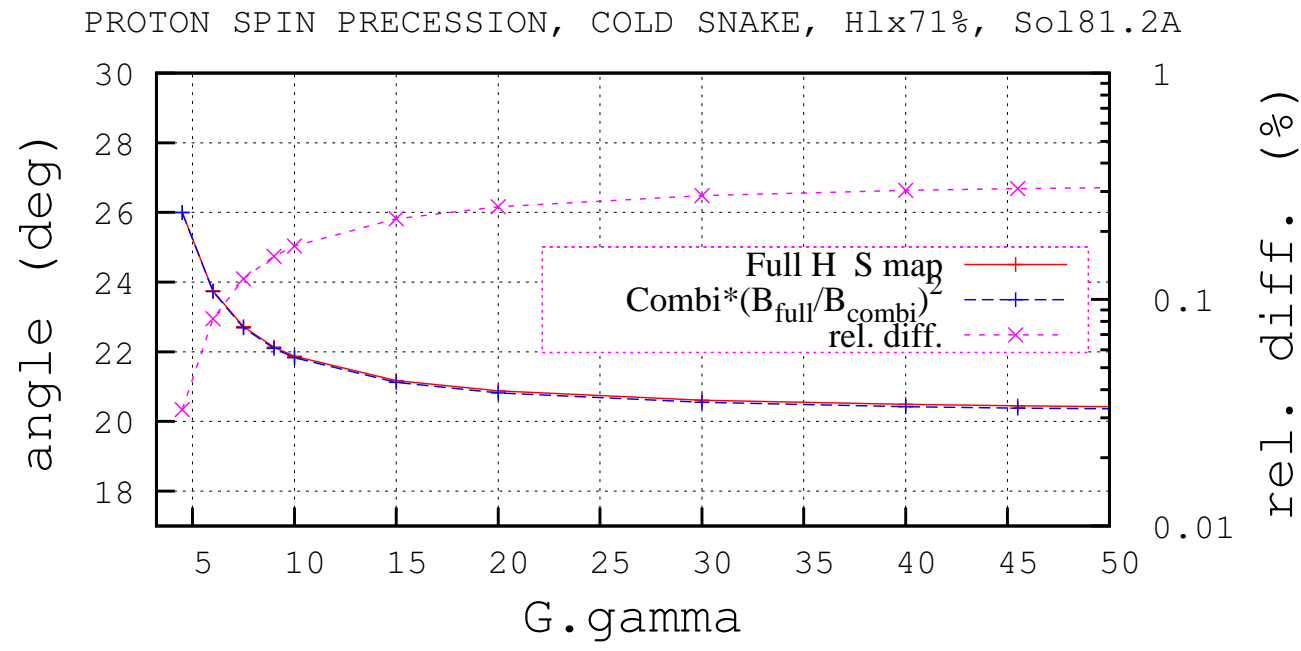

Figure 18: Proton spin precession angle from the combination of the helix and solenoid maps ("Combi" curve) and from the full helix+solenoid field map ("Full map" curve). The combined map $|B|$ value $(2.225 \mathrm{~T})$ is normalized to the full map one $(2.258 \mathrm{~T})$, an empirical factor 1.016 . The right vertical axis shows the remaining relative difference in precession angle once this re-normalization has been done, showing a maximum $0.6 \%$ at lowest $G \gamma$. 
computed independently, given a correction on the field amplitude, Figs. 18, 22 :

combined map field has to be multiplied by 1.016, in order to get agreement, on spin precession, with full map field

at better than $0.6 \%$, over the $G \gamma: 4.5 \rightarrow 50$ range, proton.

That combination appears to apply over a large range of helix and solenoid fields, including regular polarized proton settings and the substantially different polarized helion settings. This is detailed in respectively Refs. [16, Sec. 4.2-4.4] (proton) and [16, Sec. 5] (helion).

The keyword 'TOSCA' in Zgoubi does allow such linear combination of an arbitrary number of field maps. It is compatible with the 'FIT' procedure [2] thus allowing a matching of the coefficients in the linear combination for various types of constraints as orbit, focusing, field integrals, etc. Details can be found in [16, Secs. 4, 5]

\section{Transport of $\vec{n}_{0}$, from H10 septum to RHIC kickers}

The results obtained so far, regarding the transport of $\vec{n}_{0}$ from the AGS H10 septum to RHIC kickers via the AtR line, are summarized here. They confirm the validity of the choice for the AGS extraction energy in the latest RHIC runs, G $\gamma=45.5$ [23]. Details can be found in a companion paper [9].

The warm snake field map "table.55.map" has been used, properties as displayed in Tab. 1. The cold snake follows the Run 12 and Run 13 settings, thus using a linear combination of $68.2 \%$ helix (238 A for a maximum $350 \mathrm{~A}$ ) and $43.4 \%$ solenoid (152 A for a $350 \mathrm{~A}$ maximum), namely (see App. C for details on the nomenclature)

$$
\begin{gathered}
G \times[A \times \text { ags-full-coilv5-x06-rerun2-x071-integral-x5y5z10mm.table } \\
+B \times \text { ags-full-sold3-only-nodal-x5y5z10mm-wasactually-integral.table }] \\
\text { with } \quad G=1.016, \quad A=0.9606, \quad B=0.434
\end{gathered}
$$

Fig. 19 shows the beam orbit in AGS at extraction, as it is used for the computation of the periodic $\vec{n}_{0}$, in presence of the G10 kicker and of the H10 septum bumps (respectively dubbed "G09" and "H11").

Fig. 20 displays the vertical projection of the stable spin direction, as obtained from stepwise ray-tracing through the AtR, at three different locations : H10 septum in the AGS, RHIC Blue ring injection kicker, RHIC Yellow ring injection kicker. It also displays the same quantities, as obtained from spinor algebra, for comparison. 


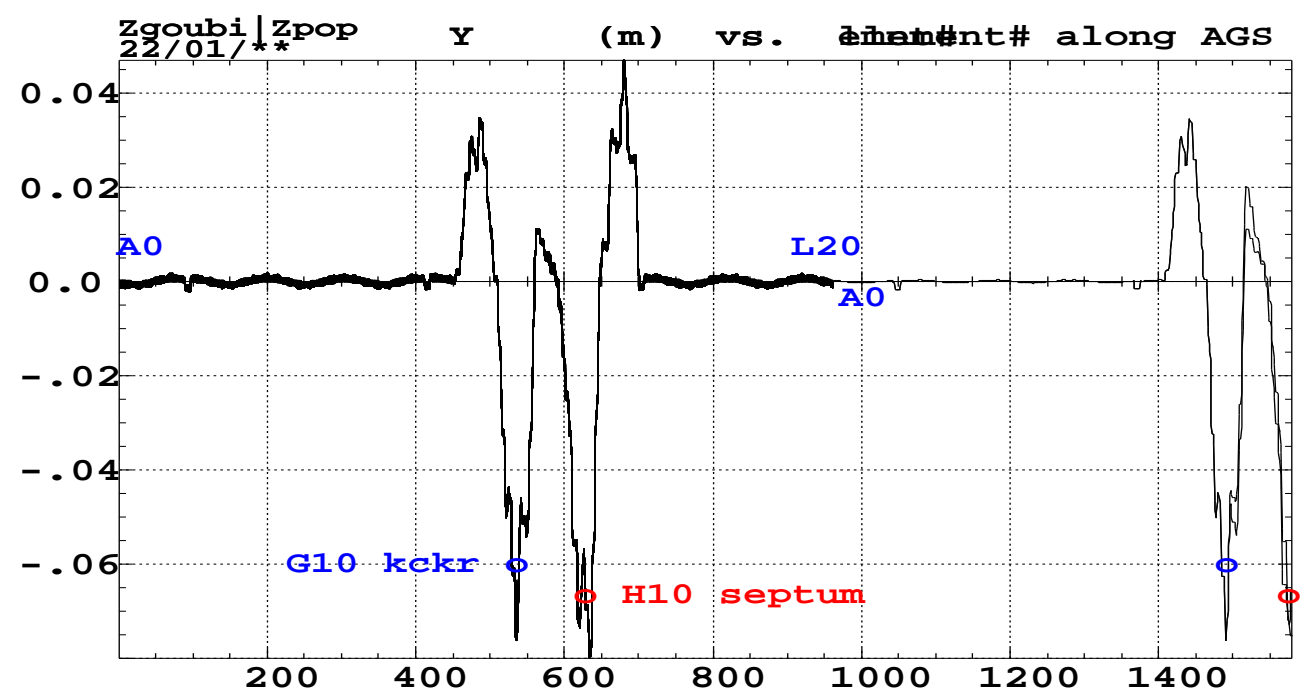

Figure 19: Beam orbit in AGS ring at extraction, from A0 to L20, including G10 kicker and H10 septum extraction bumps, followed by a final ring section from A0 to exit of H10 septum.
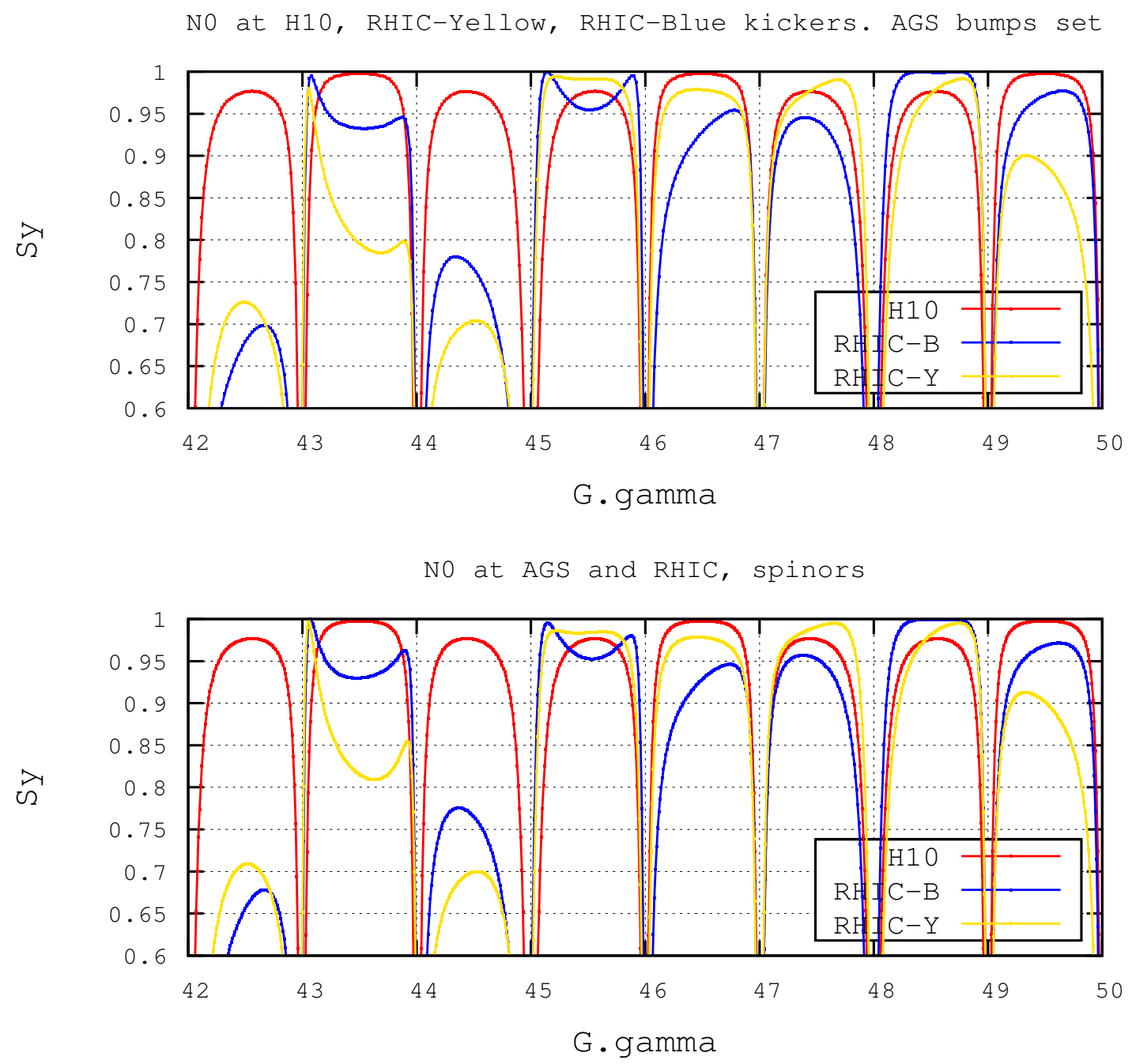

Figure 20: Vertical component of the stable spin direction, $\vec{n}_{0}$, as a function of $G \gamma$, as observed at $\mathrm{H} 10$ in the AGS, at RHIC-Blue and at RHIC-Yellow injection kickers. Top : from Zgoubi ray-tracing, bottom : from spinor algebra. 


\section{Helion}

Helion settings differ from proton due to the different anomalous magnetic moment, $\mathrm{G}=-4.18$. Present plans foresee $15 \%$ snake strength in both warm and cold snakes as a possibility. Such value requires substantially different fields, compared to proton settings.

The following investigates again, what comes out of the combined field map used in the previous sections, at such substantial distance from the design helix field (71\%), compared to a full 3-D OPERA computation with the nominal $54 \%$ helix field for $15 \%$ strength, helion.

The combined case uses (see App. $\mathrm{C}$ for details on the nomenclature)

$$
\begin{aligned}
& G \times[A \times \text { ags-full-coilv5-x06-rerun2-x071-integral-x5y5z10mm.table } \\
& +B \times \text { ags-full-sold3-only-nodal-x5y5z10mm-wasactually-integral.table }]
\end{aligned}
$$

with a global scaling factor $\mathrm{G}=1$ to start with, $\mathrm{A}=0.7602$ so yielding a $54 \%$ helix field, and $\mathrm{B}=0.228$ yielding a solenoid current $350 \times 0.228=$ $79.8 \mathrm{Amp}$ that compensates the longitudinal field on the helical orbit at $G \gamma=9$. From that combination, field modulus at magnet center comes out to be 1.6942 Tesla.

On the other hand, a newly computed OPERA full map, 54\% helix and 79.6 Amp solenoid current,

$$
\text { ags-helical-solenoid-may2013-coilv5-x054-sol-79_8A-integral-x5y5z10mm.table }
$$

shows a field modulus at magnet center of 1.7207 Tesla, in a ratio of 1.7207/1.6942=1.016 again with the combined maps field, as was found earlier with proton settings (Sec. 3).

The spin precession is interpolated at better than $0.6 \%$ accuracy (relative to tracking outcomes from the full map with field normalized to the combined case, $1.6942 \mathrm{~T}$ ), from $G \gamma=7.5$ and beyond, and with $B$ in the vicinity of $B_{0}=1.6942$ Tesla, by, Fig. 22,

$$
\mu(B, G \gamma)[\text { deg. }]=(B / B 0)^{2} *\left[26.931-3.2055 /(G \gamma)-116.708 /(G \gamma)^{2}-4171.7 /(G \gamma)^{3}\right]
$$

Additional details concerning this study can be found in Ref. [16, Sec. 5]. 


\section{Cold snake, Helion settings. Motion, field, spin precession}
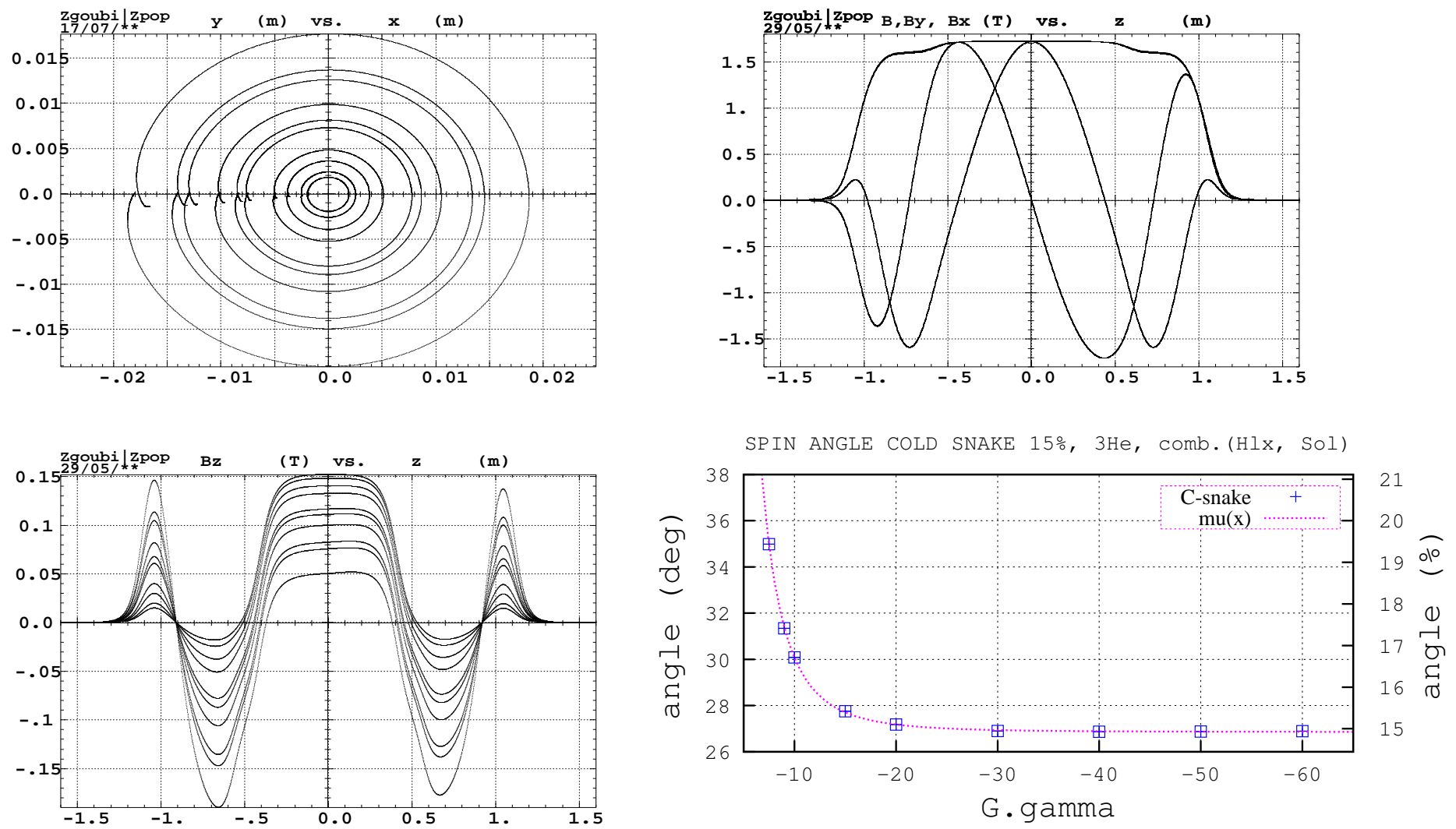

Figure 21: Top left : projection of helical orbits with $G \gamma \in[7.5,60]$ onto the transverse plane $(x, y)$. Top right : transverse field components along these orbits. Bottom left : longitudinal field components along the orbits. Bottom right : spin precession from tracking (markers) and interpolation function, Eq. 6.

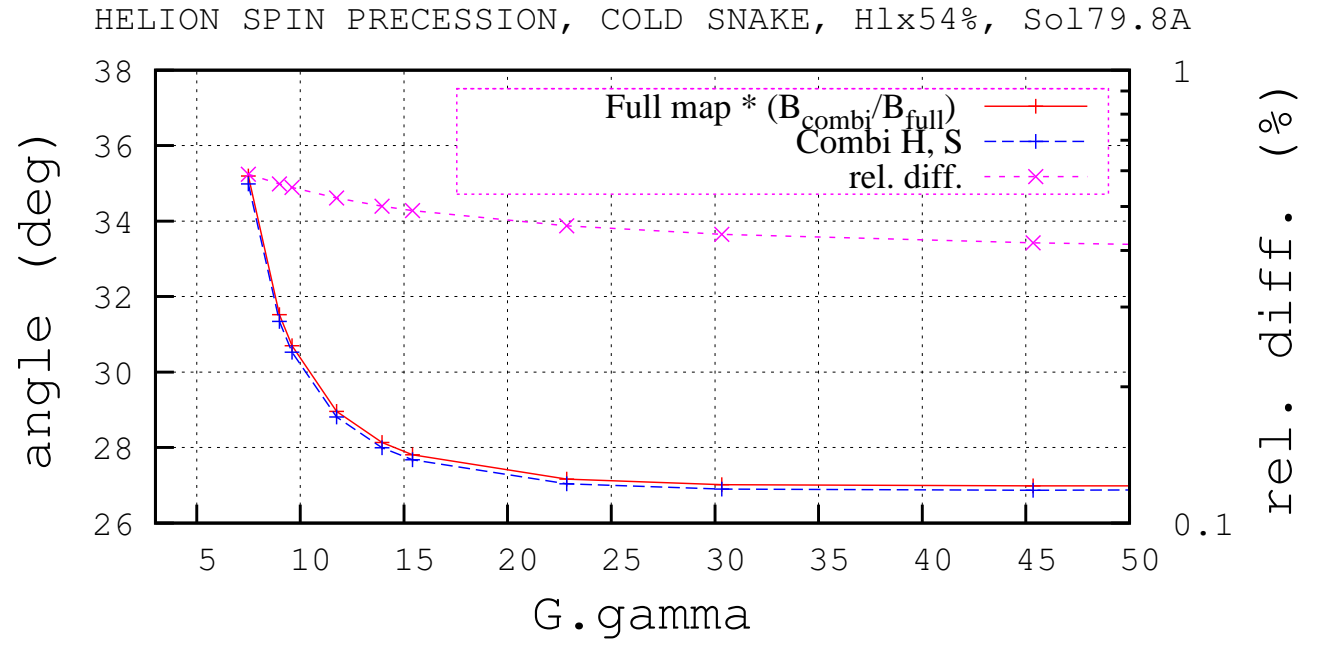

Figure 22: Helion spin precession angle from the combined helix+solenoid field map ("Combi H, S" curve) and from the full helix and solenoid field map ("Full map" curve). The full map $|B|$ value (1.72 T) has been normalized to the combined maps one (1.694 T), an empirical factor of 1.016. The right vertical axis shows the remaining relative difference in precession angle once this re-normalization has been done, showing a maximum $0.6 \%$ at lowest $G \gamma$. 


\section{Conclusion}

The AGS warm and cold snake field maps have been re-visited, many comparisons have been performed with earlier works as published in C-AD Tech. Notes [10]-[15], they are reported in detail in Ref. [16].

The results so obtained have been summarized in the present Tech. Note.

This study has resulted in a series of findings, as follows.

- in the case of the warm snake field map, CA-AP Note 136 gives a snake strength in the low rigidity region which is larger than what is obtained today using (i) the same field map and (ii) two different tracking codes, Raytrace [26] and Zgoubi, which do agree with each other. The difference observed in the computed snake strength increases with decreasing rigidity, Fig. 23 and Tab. 3 in App. A.

- same observation for the cold snake field map case, CA-AP Note 128 and Ref. [11] give a snake strength in the low rigidity region which is larger than what is obtained today, using the same field maps and either Raytrace or Zgoubi, which again agree with each other, Fig. 25 and Table 4 in App. B.

- today's data derived from 3-D field maps using Zgoubi provide optical parameters (first order mapping and other orbit excursions in the snakes) in good agreement with tracking outcomes found in Ref. [15] as to the warm snake and in Ref. [10,12] as to the cold snake.

- the compensation solenoid in the cold snake has been operated up to now with a current of 152 Amps. It appears that the required field, in order to compensate the longitudinal helix field component at $G \gamma=9$ with a $68.2 \%$ helix, is actually 76 Amps, half the Run 13 value,

- a change in solenoid setting is not innocent in terms of spin precession, it changes snake strength at all energies, compare Figs. 12 and 17 an cols. 4-6 in Tab. 1 ,

- it has been established for the cases studied that a global scaling factor of about 1.016 has to be applied, when fabricating a map by linear combination of separate pure helix and pure solenoid OPERA field maps, for solenoid fields in 80 150 Amp range, in order to restore the optics and spin precession resulting from a complete helix+solenoid OPERA computation, Secs. 2.3.2, 3, 5. That 1.016 correction factor was found to be valid even for a large change in the helix field, from proton ( $71 \%$ helix) to helion ( $54 \%$ helix), Secs. $3,5$.

Besides, it is planned to re-compute warm snake field maps, including different current settings in view of further proton and helion spin dynamics studies. The first order matrices in the MADX model of the AGS will be re-computed using these new sets of OPERA maps and the ray-tracing code Zgoubi.

The computation of new field maps is undertaken bearing in mind that long-term multi-turn 9-D tracking of particle and spin motion require finer mesh and longer extent than the old field maps provide, in order to ensure highest accuracy of field interpolation from the 3-D mesh. This is necessary for a sound use of the stepwise ray-tracing method to model the AGS ring, especially in the low-energy part of the acceleration cycle where the helical orbit in the snakes reaches a radius of $15-25 \mathrm{~mm}$.

\section{Acknowledgements}

With thank M. Okamura and J. Takano (KEK) for various discussions and for providing us with OPERA files of the warm snake. 


\section{APPENDIX}

\section{A Spin precession in warm snake}

Discrepancies with earlier published data, found during the present study, are discussed here.

In Fig. 23,

- the "AP\#136" curve is from Ref. [15], representing $\mu(B, \gamma)=\left(\frac{B}{B_{0}}\right)^{2}\left(C 1+C 2 / \gamma^{2}\right)$, with B=B0, C1 $=10.453 \mathrm{C} 2=27.673$. Snake strength in CA-AP\#136 was computed using SNIG [27] and OPERA 3-D field maps.

See also C-AD MAC, Nov. 8-9 2004, AGS Cold Snake Commissioning slides, where one finds :

"New warm helical snake, B=1.53 T, strength 8.3\% (15 deg.) at injection, 5.9\% (10.6 deg) at extraction, data obtained using "SNIG" code tracking in OPERA field map. “

- whereas the "Fit" curve, on top of Zgoubi tracking data (markers, "W-snake"), uses $\mu(B, \gamma)=(B / B 0)^{2}\left(C 1+C 12 / \gamma+C 2 / \gamma^{2}\right)$, taking $\mathrm{B}=\mathrm{B} 0, \mathrm{C} 1=10.58413, \mathrm{C} 12=-0.816718, \mathrm{C} 2=11.0849$. Snake strength $6.7 \%$ at injection, $5.9 \%$ at extraction.

"C-AD Note 136" precession and orbit data values in Tab. 3 are from Tables 1 and 2 therein. By contrast with spin precession, the agreement with Zgoubi data as to orbit geometry is good, at all $G \gamma$, cols. 6-8 in Tab. 3.

Table 3: Spin precession in the warm snake, detailed data from tracking. In col. 4, spin precession data from CA-AP\#136 (black numbers) can be compared with Zgoubi outcomes (blue numbers). The difference increases with decreasing rigidity. Cols. 6-8 compare paraxial transport data, the agreement is good.

\begin{tabular}{|c|c|c|c|c|c|c|c|}
\hline \multirow[b]{2}{*}{$B \rho / B \rho(G \gamma=4.5)$} & \multirow[b]{2}{*}{$G \gamma$} & \multirow[b]{2}{*}{$\gamma$} & \multirow[b]{2}{*}{$\begin{array}{c}\mu(\mathrm{deg}) \\
\text { C-AD Note } 136 \\
\text { Zgoubi }\end{array}$} & \multirow[b]{2}{*}{$\begin{array}{c}\mu \\
(\%)\end{array}$} & \multicolumn{3}{|c|}{ geometry is ok! } \\
\hline & & & & & $\begin{array}{c}\mathrm{ds} \\
(\mathrm{mm})\end{array}$ & $\begin{array}{c}\max x \\
(\mathrm{~mm})\end{array}$ & $\begin{array}{r}\max y \\
(\mathrm{~mm})\end{array}$ \\
\hline 0.99527469 & 4.48211837 & 2.5 & $\begin{array}{l}15.06029 \\
12.03852\end{array}$ & $\begin{array}{l}8.37 \\
6.69\end{array}$ & $\begin{array}{l}3.4638 \\
3.4534\end{array}$ & $\begin{array}{c}18.7709 \\
18.56\end{array}$ & $\begin{array}{l}19.45 \\
19.34\end{array}$ \\
\hline 1.502203956 & 6.45425046 & 3.6 & $\begin{array}{c}12.345959 \\
11.19923\end{array}$ & & $\begin{array}{l}1.5114 \\
1.5071\end{array}$ & $\begin{array}{c}12.458 \\
12.26\end{array}$ & $\begin{array}{l}12.85 \\
12.75\end{array}$ \\
\hline 2.481637751 & 10.39851463 & 5.8 & 11.184056 & & & & \\
\hline 3.447732692 & 14.3427788 & 8 & $\begin{array}{c}10.884765 \\
10.6719\end{array}$ & & $\begin{array}{c}0.286 \\
0.29\end{array}$ & $\begin{array}{l}5.35 \\
5.33\end{array}$ & $\begin{array}{l}5.59 \\
5.55\end{array}$ \\
\hline 4.540190075 & 18.82489717 & 10.5 & 10.752696 & & & & \\
\hline 8.676603671 & 35.856947 & 20 & 10.62500 & & & & \\
\hline 10.85064630 & 44.82118375 & 25 & $\begin{array}{c}10.607894 \\
10.56448\end{array}$ & $\begin{array}{l}5.89 \\
5.87\end{array}$ & $\begin{array}{l}0.02883 \\
0.02875\end{array}$ & $\begin{array}{c}1.7180 \\
1.693\end{array}$ & $\begin{array}{l}1.7764 \\
1.7625\end{array}$ \\
\hline 11.01524059 & 45.5 & 25.3786 & 10.56092 & & 0.0160 & & \\
\hline
\end{tabular}

Comparison with data from Raytrace - Essentially the numerical integrator in the ray-tracing code Raytrace (RK4) differs with Zgoubi method (Taylor series [2]).

The same 3-D map, "table.55.map", is used with the two codes. The same initial coordinates (that yield helix centered on snake axis, Fig. 2) are taken. Fig. 24 shows the agreement between the two codes, at better than $1 \%$ level. 


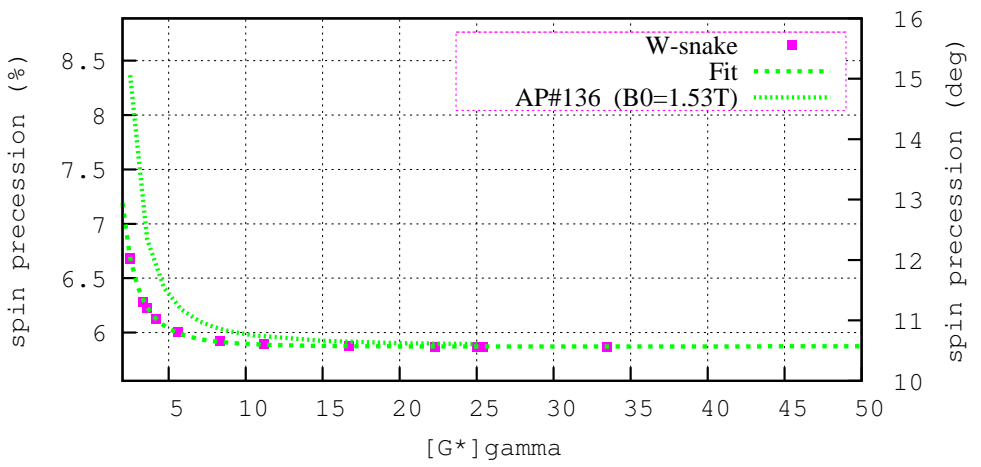

Figure 23: Spin precession discrepancy, warm snake (a plot of the data in Tab. 3).

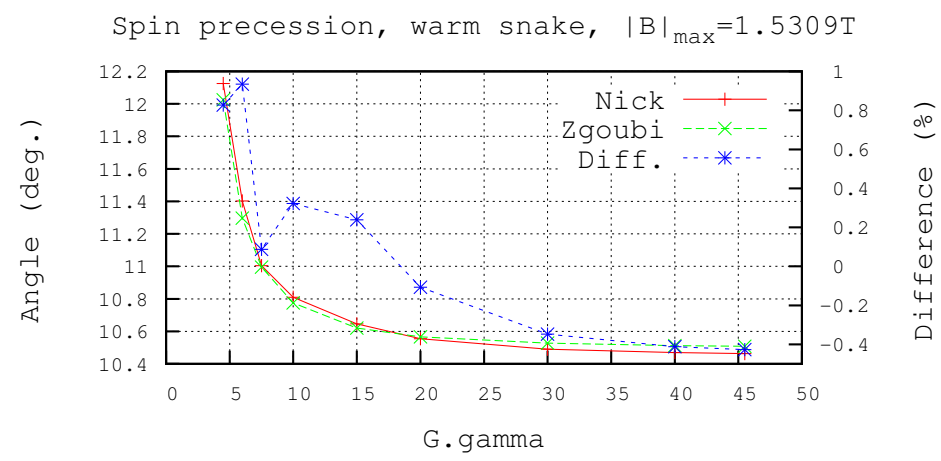

Figure 24: Warm snake. This plot shows that Raytrace data confirm Zgoubi ones.

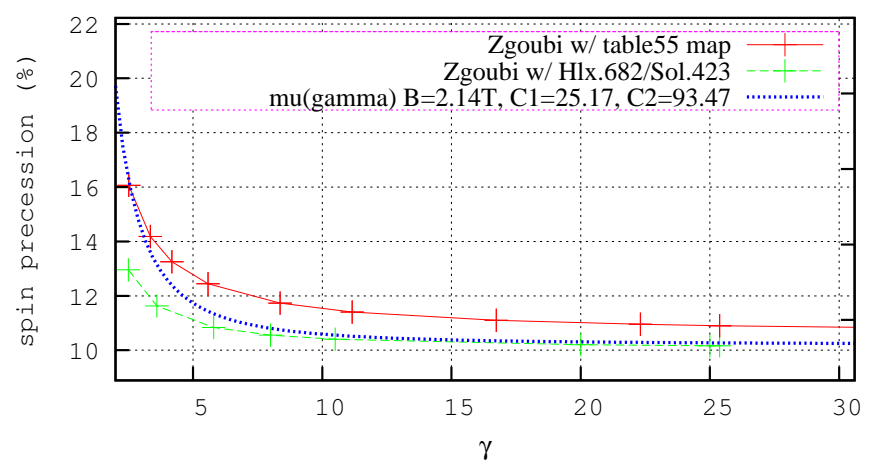

40

35 ठำ

30 -

25

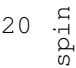

Figure 25: Spin precession discrepancy, cold snake (a plot of the data in Tab. 4).

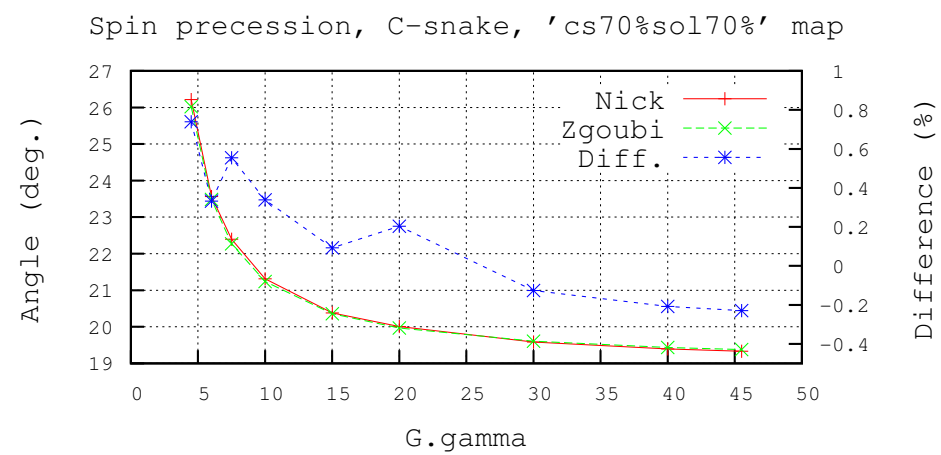

Figure 26: Cold snake. This plot shows the general agreement between Raytrace and Zgoubi outcomes. 


\section{B Spin precession in cold snake}

Discrepancies with earlier published data, found during the present study, are discussed here.

Three of the original field maps of the cold snake are available,

- a full map of the helix+solenoid magnet, "ref+sole.tab",

- two independent maps, pure helix and pure solenoid, respectively "table_for_Helix_3T.tab", "table_for_Solen_3T.tab". The latter had been obtained by substation of the former (obtained from a dedicated OPERA run) from a full 3-D field map of the helix+solenoid magnet.

Field integral, in the case "ref+sole.tab" map : $\int_{\text {path }} B d s=4.38827$ T.m. Plateau is at B=2.20 T.

Taking Run 13 settings instead, using the other two maps, helix 68.2\% (238 Amp) + solenoid 42.3\% (151.5 Amp): $\int_{\text {path }} B d s=$ 4.3014765 T.m. Plateau is at $\mathrm{B}=2.14 \mathrm{~T}$.

Note : this comparison of the two maps, full and combined, does not discuss the unexpected, unphysical, field data further obtained from "ref+sole.tab", details in Ref. [16, Sec. 2.2].

Table 4: Spin precession in the warm snake, detailed data from tracking.

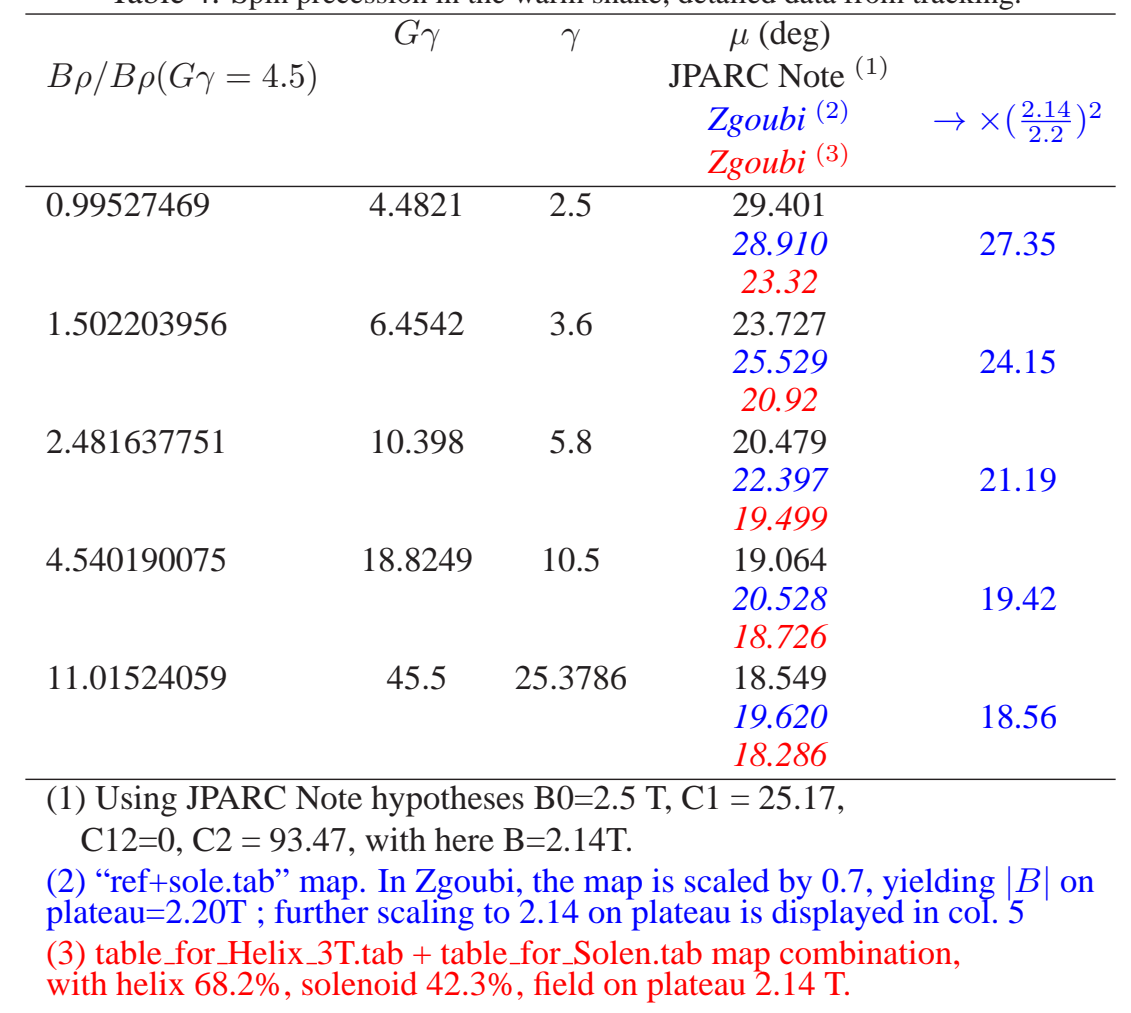

Comparisons with Raytrace data - The same, 3-D maps are used with the two codes. The same initial coordinates (that yield helix centered on snake axis) are taken. Fig. 26 shows the agreement between the two codes.

\section{Cold snake, linear combination of helix and solenoid field maps}

The 'TOSCA' procedure in Zgoubi allows combining several 3-D field maps, with each one its scaling coefficient, and applying an additional global scaling coefficient to the result. For instance, in the present case of concern of a pair of helix and solenoid field maps, that linear combination takes the form

$$
\begin{array}{r}
G \times[A \times \text { ags-full-coilv5-x06-rerun2-x071-integral-x5y5z10mm.table } \\
\oplus B \times \text { ags-full-sold3-only-nodal-x5y5z10mm-wasactually-integral.table }]
\end{array}
$$

A "principle of linear combination" was first assessed [16] by using the pure helix map with $71 \%$ current on the one hand (A=1, Eq. 7), and on the other hand the pure solenoid map with current 152Amp / 350Amp $=43.4 \%(B=0.434)$. These conditions are those of the following full 3-D field map, used for comparison in that assessment :

$$
\text { ags-full-coilv5-x06-rerun2-x071-sol-152a-nodal-x5y5z10mm.table }
$$

The tracking outcomes of that full map are those displayed in col. 4 of Tab 1. 
Note that, for that particular setting, helix $71 \%$ and solenoid $43.4 \%$, the field integrals $\int B_{z} d s$ on trajectory come out to be :

\begin{tabular}{l|c}
$G \gamma$ & $\int B_{z} d s$ (T.m) \\
\hline 7.5 & $9.18407052 \mathrm{E}-02$ \\
10 & $1.35670277 \mathrm{E}-01$ \\
45.5 & $2.32933972 \mathrm{E}-01$
\end{tabular} .

It results from tracking in the linear combination of the two maps that the integral at $G \gamma=9$ can be cancelled using instead $23.2 \%$ solenoid field (81.20 Amp), B=0.232 in Eq. 7. Following that change in B (from 0.434 to 0.232 ) a difference in field modulus appears between the combined map and the full 3-D one, 2.2584 Tesla in the former, versus 2.2252 Tesla in the latter, hence a necessary correction factor (the global $\mathrm{G}$ factor, Eq. 7) $G=2.2584 / 2.2252=1.016$ for the former to be a better approximation of the latter.

In matter of spin precession, this normalization of the combined map's $|B|$ value to the full map's one (or the other way) by that 1.016 factor, results in a marginal residual difference in spin precession angle, as displayed in Fig. 18 (proton) and Fig. 22 (helion).

\section{Cold snake, cancelling $\int B_{z} d s$ in presence of $68.2 \%$ or $71 \%$ helix fields}

The use of combined helix+solenoid field maps, and in particular a $\mathrm{G}=1.016$ global field scaling factor (Eq. 7) as introduced below, are discussed in Sec. 3 and in App. C.

In order to represent the cold snake in the case of a $68.2 \%$ helix and solenoid current set to cancel $\int B_{z} d s$, the combination in Zgoubi takes the form (using the notations in Eq. 7)

$$
\begin{aligned}
1.016 & \times[0.9606 \times \text { ags-full-coilv5-x06-rerun2-x071-integral-x5y5z10mm.table } \\
\text { B } & \times \text { ags-full-sold3-only-nodal-x5y5z10mm-wasactually-integral.table }]
\end{aligned}
$$

given $A=68.2 / 71=0.9606$ helix field scaling. Tracking yields $B=0.2172$ solenoid field scaling $(0.2172 \times 350=76$ Amp. $)$ so to cancel $\int B_{z} d s$ at $G \gamma=9$.

In order to represent the cold snake in the case of a $71 \%$ helix and solenoid current set to cancel $\int B_{z} d s$, the combination takes the form (using the notations in Eq. 7)

$$
\begin{aligned}
& 1.016 \times[\text { ags-full-coilv5-x06-rerun2-x071-integral-x5y5z10mm.table } \\
& \text { B } \times \text { ags-full-sold3-only-nodal-x5y5z10mm-wasactually-integral.table }
\end{aligned}
$$

given $A=1$ helix field scaling. Tracking yields $B=0.2357$ solenoid field scaling $(0.2357 \times 350=82.5$ Amp. $)$ so to cancel $\int B_{z} d s$ at $G \gamma=9$.

\section{Two remarks}

(i) The solenoid currents are in a ratio $0.2172 / 0.2357 \approx 0.922$ which identifies with the squared ratio of the helix fields $\times(68.2 / 71)^{2} \approx 0.923$, the reason is discussed in Sec. 2.3.2.

(ii) The solenoid current in the $71 \%$ helix case here, $82.5 \mathrm{Amp}$, has been worked out more precisely, this is the reason for a slight difference, $+1.6 \%$, with the value used in the text, 81.2 Amp. Tracking shows that the former value yields $\int B_{z} d s=1.810^{-5}$ T.m at $G \gamma=9$, whereas the latter yields $\int B_{z} d s=-2.310^{-3}$ T.m. Conclusion : the second integral is anyway close enough to zero that the goal the ensuing effect on coupling in the AGS can be neglected. 


\section{References}

[1] Modelling of the AGS using the ray-tracing code Zgoubi, see PAC and IPAC conference papers, F. Méot et al., 2009 and beyond, and also, Spin tracking simulations in AGS based on ray-tracing methods, F. Méot et al., Tech. Note C-AD/AP/452 (Sept. 2009). Zgoubi-ing AGS : spin motion with snakes and jump-quads, F. Méot et al., Tech. Note C-AD/AP/453 (Dec. 2009).

[2] Zgoubi Users' Guide, F. Méot, CA/AP/470, Oct. 2012.

Source code, examples and Guide down-loadable at http://www.scienceaccelerator.gov/dsa/result-list/fullRecord:zgoubi/.

More documentation at http://sourceforge.net/projects/zgoubi/.

[3] Modelling of the AGS using Zgoubi - status, F. Méot et al., Procs. IPAC 2011 Acc. Conf.

[4] Helical partial snake for the AGS, T. Roser, et al., RHIC Tech. Note SN072, March 17, 1998.

[5] Acceleration of polarized protons in the AGS with two helical partial snakes, H. Huang et al., Procs. EPAC 2006 Acc. Conf., Edinburgh.

[6] Towards full preservation of polarization of proton beam in the AGS, F. Lin, PhD Thesis, AGS and Indiana University, 2007.

[7] Increasing the AGS Beam Polarization with 80 Tune Jumps, V. Schoefer et al., http://accelconf.web.cern.ch/AccelConf/IPAC2012/papers/tuxa03.pdf

[8] Stable spin direction [...] at the injection point of RHIC, N. Tsoupas et al., Spin Note AGS/RHIC/SN 21 (1996).

[9] Where is $\vec{n}_{0}$ ?, F. Méot, H. Huang, N. Tsoupas, CA-AP Note, to be released (2013).

[10] Cold AGS snake optimization by modeling, A.U. Luccio et al., CA/AP/128, Dec. 2003.

[11] Tracking of polarized protons in the main ring of the J-PARC accelerator facility, A.U. Luccio, KEK report, Jan. 4, 2006.

[12] Matching the Cold AGS Snake to the AGS Lattice, A. Luccio et al., C-A/AP/167 (2004).

[13] Focusing of the AGS Cold Snake, A. Luccio et al., C-A/AP/248 (2006).

[14] Calculation of the focusing properties of a 7\% partial snake [...], N. Tsoupas et al., C-A/AP/134 (2004).

[15] Tracking through a warm helical snake for the AGS, A. Luccio et al., C-A/AP/136 (2004).

[16] /rap/lattice_tools/zgoubi/AgsZgoubiModel/snakeFieldMaps/rotationAngle/Log_130103/slides.pdf

[17] Field measurements in the AGS warm snake, J. Takano et al., Procs. EPAC 2004 Acc. Conf., Lucerne.

[18] RHIC injector complex online model status and plans, V. Schoefer et al., Proc. PAC09 Acc. Conf., Vamcouver.

[19] Superconducting helical snake magnet for the AGS, E. Willen et al., Procs. PAC 2005 Acc. Conf., Knoxville.

[20] Magnetic design of a superconducting AGS snake, R. Gupta et al., Procs. PAC 2003 Conf., Portland.

[21] Orbits and field in the helical wiggler, J. P. Blewett, R. Chasman, J. App. Phys, Vol. 48, No. 7, July 1977.

[22] Handbook of Accelerator Physics and Engineering, A. Chao, M. Tigner, World Scientific.

[23] Spin matching from AGS to RHIC with two partial snakes, W. W. MacKay et al., RHIC Tech Note.

[24] Transport of polarized ${ }^{3}$ He in Booster and AGS, F. Méot, Workshop on Opportunities for Polarized He-3 in RHIC and EIC, BNL, Sept. 2011.

[25] Stable spin direction of a polarized proton beam at the injection point of RHIC, N. Tsoupas et al., Spin Note AGS/RHIC/SN 021, March 1996.

[26] The ion-optical program Raytrace, S. B. Kowalski, H. A. Enge, NIM A258 (1987) p.407.

[27] A.U. Luccio, Technical Report AGS/RHIC/SN No.03 BNL (1996) 